\title{
VARIATIONS IN THE AGRONOMIC AND QUALITY CHARACTERISTICS OF DOMESTIC AND FOREIGN SAFFLOWER (Carthamus tinctorius L.) GENOTYPES
}

\author{
Sabri ERBAS*, Muhammet TONGUC, Arif SANLI \\ Suleyman Demirel University, Faculty of Agriculture, Department of Field Crops, Isparta, TURKEY \\ *Corresponding author: sabrierbas@sdu.edu.tr
}

Received: 25.01.2016

\begin{abstract}
The present study was carried out to determine the agronomic and quality characteristics of domestic and foreign safflower (Carthamus tinctorius L.) genotypes in the 2011-2012 growing season in Isparta. In the study, 39 safflower genotypes were used as the plant material. The highest 1000 seed weight and hull content were detected in Ziyang. Arizona SC III was determined as the genotype with the lowest 1000 seed weight, hull content, seed yield, and oil yield. The highest seed yield was recorded in UC-1 (215.9 kg da-1). Oil contents of the genotypes ranged from 22.6 to $33.8 \%$ on average and Arizona SC III, Oleic Leed, Centennial, Finch, Ole, S-517, Enana and Leed were determined as the genotypes with the highest oil content. Variation for oleic acid was between 11.1 and $68.3 \%$ in 2011 and between 12.0 and $71.6 \%$ in 2012 . Linoleic acid content varied between 18.0 and $74.7 \%$ in 2011 and between 14.2 and 73.9\% in 2012. Montola 2000, Ole, UC-1, NO 55-663 and $\mathrm{S}-517$ were found to contain more than $60 \%$ oleic acid. Both performances of the genotypes used in the study could further examined by cultivating in different locations and a successful hybridization program compatible with the desired goals of breeding may be drawn up using wide variations reported among the examined morphological and agronomic characters.
\end{abstract}

Keywords: adaptation, Carthamus tinctorius, Safflower, yield and oil composition

\section{INTRODUCTION}

Safflower (Carthamus tinctorius L., Compositeae) is an important oilseed crop which began to be cultivated in the Middle East 3000 years ago. Approximately 647.000 tons of safflower is produced in an area of 782.000 ha worldwide (Anonymous, 2015a). India, USA, Mexico, Ethiopia and Argentina account for approximately $95 \%$ of the world's safflower production. Some 70.000 tons of safflower seeds are produced in an area of 43.107 ha in Turkey in 2015 (Anonymous, 2015b). The production of oilseed crops is not at sufficient level in Turkey to meet edible oil demand; therefore large imports of both seed and oil are necessary. The annual oil seed production of Turkey was 2.7 million tons in 2014. Turkey imported 6.3 million tons of vegetable oil, oil seeds, and oil-cakes in total, which amounted to $\$ 4.3$ billion-3.1 million tons of oil seeds ( $\$ 1.8$ billion), 1.6 million tons of crude oil (\$1.9 billion), and 1.6 million tons of oil-cakes ( $\$ 597$ million) in 2014 (Anonymous, 2015a).

In comparison with other oilseed crops, safflower is a crop with high adaptation ability in regions where winter months are cool and summer months are arid. Especially due to its high tolerance for aridity and moderate tolerance to the cold, it is one of the alternative crops to be utilized in the arid and semi-arid agricultural lands of Turkey, particularly in Eastern Anatolia, South-eastern Anatolia, and Central Anatolia Regions (Baydar and Erbas, 2007; Öztürk, 2008). Even though genotypes with high oil content (above 40\%) have been identified thorough germplasm screenings, the oil content of the current safflower cultivars remains far below this level (Johnson et al., 1999). To obtain crops from safflower in economic terms and in order for them to compete with other oil seed crops such as; sunflower, soybean, and canola, cultivars with high oil content and seed yield should be developed and cultivated (Pahlavani 2005). Thus increased seed yield and oil content are two important aims of the most of the safflower breeding programs.

Besides cultural treatments, the identification and selection of appropriate cultivars is also essential for safflower cultivation. The proper selection of those cultivars which will particularly be able to adapt to dry land conditions will contribute to the obtaining competitive yields to cultivate safflower economically. Therefore, in order to determine the cultivars suitable for a region, it is important to use a large number of genotypes to establish their agronomic and quality characters through adaptation experiments. Various researchers reported that the growth of cultivars varied by arid and low land 
conditions and that irrigation and rainfall increased the seed yield at the stages of branching and seed formation; furthermore, yield and crop traits were shown to vary by cultivar, ecological conditions, and agronomic practices. The large number of genotypes allows observation of exiting genetic variation which could be used to develop hybridization programs among the genotypes for the development of new cultivars with high yield potential, high oil content, and different fatty acid compositions (Mary and Gopalan 2006; Karademir et al. 2007). In the present study, safflower genotypes originated from different countries were evaluated for their adaptation ability in Isparta ecological condition to reveal suitable types for further studies.

\section{MATERIALS AND METHODS}

In this study, 39 safflower genotypes were used as the plant material. Genotypes of Turkish origin (Remzibey05, Dincer 5-118, and Yenice 5-38) were obtained from Ege Agricultural Research Institute, and the other genotypes were provided by USDA Western Regional PI Station in Pullman, WA, USA (Table 1).

Table 1. Safflower genotypes used in the study as the plant material

\begin{tabular}{|c|c|c|c|c|c|c|c|c|c|}
\hline $\begin{array}{l}\text { Gen Bank } \\
\text { No }\end{array}$ & Genotype & Phenotype* & Origin & Registration & Gen Bank No & Genotype & Phenotype & Origin & Registration \\
\hline PI 537110 & Quiriego 88 & $\mathrm{Y}-\mathrm{S}$ & Mexico & Cultivar & PI 538779 & Centennial & $\mathrm{Y}-\mathrm{S}$ & USA & Cultivar \\
\hline PI 537111 & Sahuaripa 88 & $\mathrm{O}-\mathrm{S}$ & Mexico & Cultivar & PI 601506 & S-517 & $\mathrm{O}-\mathrm{S}$ & USA & Cultivar \\
\hline PI 561703 & San Jose 89 & $\mathrm{O}-\mathrm{S}$ & Mexico & Cultivar & PI 572472 & Rehbein & Y-S & USA & Cultivar \\
\hline PI 572475 & Saffire & $\mathrm{R}-\mathrm{S}$ & Canada & Cultivar & PI 525458 & Finch & $\mathrm{Y}-\mathrm{S}$ & USA & Cultivar \\
\hline PI 592391 & AC Sunset & $\mathrm{R}-\mathrm{S}$ & Canada & Cultivar & PI 572436 & Leed & $\mathrm{O}-\mathrm{S}$ & USA & Cultivar \\
\hline PI 559909 & AC Stirling & Y-S & Canada & Cultivar & PI 572415 & No 55-633 & $\mathrm{O}-\mathrm{S}$ & USA & Cultivar \\
\hline PI 603206 & Lesaf 414 & $\mathrm{R}-\mathrm{S}$ & Canada & Pure line & PI 508098 & Hartman & Y-S & USA & Cultivar \\
\hline PI 610263 & Enana & $\mathrm{O}-\mathrm{S}$ & Spain & Pure line & PI 537695 & Ole & $\mathrm{O}-\mathrm{S}$ & USA & Cultivar \\
\hline W6 16828 & Rinconada & $Y-S$ & Spain & Cultivar & PI 572434 & UC-1 & $Y-S$ & USA & Cultivar \\
\hline W6 16833 & $\mathrm{CH}-353$ & Y-S & Spain & Pure line & PI 572414 & US-10 & Y-S & USA & Cultivar \\
\hline TR 69497 & Dincer 5-118 & O-S1 & Turkey & Cultivar & PI 572471 & Sidwill & Y-S & USA & Cultivar \\
\hline TR 69498 & Yenice 5-38 & R-S1 & Turkey & Cultivar & PI 537694 & Royal & $\mathrm{O}-\mathrm{S}$ & USA & Cultivar \\
\hline TR 69499 & Remzibey-05 & Y-S & Turkey & Cultivar & PI 537692 & Gila & $\mathrm{O}-\mathrm{S}$ & USA & Cultivar \\
\hline PI 538025 & Montola 2000 & Y-S & USA & Cultivar & PI 514632 & Ziyang & R-S & China & Cultivar \\
\hline PI 601166 & Oker & $\mathrm{O}-\mathrm{S}$ & USA & Cultivar & PI 514631 & Yuyao & $\mathrm{R}-\mathrm{S}$ & China & Cultivar \\
\hline PI 572465 & 4022 & $\mathrm{R}-\mathrm{S}$ & USA & Pure line & PI 514624 & Shufu & $\mathrm{R}-\mathrm{S}$ & China & Cultivar \\
\hline PI 572439 & PCA & Y-S & USA & Pure line & PI 514620 & Huaxian & $\mathrm{R}-\mathrm{S}$ & China & Cultivar \\
\hline PI 572418 & Arizona SC III & W-S & USA & Pure line & PI 506426 & FO-2 & $\mathrm{R}-\mathrm{S}$ & China & Cultivar \\
\hline PI 572421 & Frio & $Y-S$ & USA & Cultivar & - & Gifford & $\mathrm{R}-\mathrm{S}$ & USA & Cultivar \\
\hline PI 560177 & Oleic Leed & $\mathrm{O}-\mathrm{S}$ & USA & Cultivar & & & & & \\
\hline
\end{tabular}

The research was conducted in Isparta $\left(37^{\circ} 45^{\prime} \mathrm{N}\right.$ and $\left.30^{\circ} 33^{\prime} \mathrm{E}, 997 \mathrm{~m}\right)$, located in the inner Mediterranean Region of Turkey, in 2011 and 2012. The major soil characteristics of the research area, based on the method described by Rowell (1996), were as follows: the soil texture was clay loam; the organic matter was $1.1 \%$ by the
Walkley-Black method; lime was $7.2 \%$ by Schiebler calcimeter; total salt was $0.38 \%$; exchangeable $\mathrm{K}$ was 119 mg kg-1 by $1 \mathrm{~N} \mathrm{NH}_{4} \mathrm{OAc}$; extractable $\mathrm{P}$ was $3.9 \mathrm{mg} \mathrm{kg}^{-1}$ by $0.5 \mathrm{~N} \mathrm{NaHCO}_{3}$ extraction; and the $\mathrm{pH}$ in a soil saturated extract was 7.5. The climatic data for the experimental area were given in Table 2 .

Table 2. Total precipitation, mean humidity and mean temperatures and long term averages of experimental site

\begin{tabular}{lccccccccc}
\hline \multirow{2}{*}{ Month } & \multicolumn{3}{c}{ Precipitation, $\mathbf{L ~ m}^{\mathbf{2}}$} & \multicolumn{3}{c}{ Mean temperature, ${ }^{\mathbf{}} \mathbf{C}$} & \multicolumn{2}{c}{ Mean humidity, \% } \\
\cline { 2 - 9 } & $\mathbf{1 9 7 5 - 2 0 1 2}$ & $\mathbf{2 0 1 1}$ & $\mathbf{2 0 1 2}$ & $\mathbf{1 9 7 5 - 2 0 1 2}$ & $\mathbf{2 0 1 1}$ & $\mathbf{2 0 1 2}$ & $\mathbf{1 9 7 5 - 2 0 1 2}$ & $\mathbf{2 0 1 1}$ & $\mathbf{2 0 1 2}$ \\
\hline March & $51.8^{*}$ & 50.4 & 21.4 & 6.1 & 6.3 & 5.2 & 65.1 & 70.2 & 58.1 \\
April & 56.1 & 42.8 & 53.4 & 10.7 & 10.3 & 11.7 & 61.1 & 68.5 & 57.6 \\
May & 46.1 & 42.5 & 108.4 & 15.5 & 14.4 & 14.5 & 58.3 & 64.8 & 65.6 \\
June & 28.3 & 61.8 & 18.2 & 20.3 & 19.8 & 22.4 & 49.9 & 56.6 & 46.1 \\
July & 12.4 & 1.8 & 0.0 & 23.7 & 25.0 & 25.4 & 44.2 & 42.3 & 42.1 \\
August & 12.4 & 0.6 & 0.2 & 23.2 & 22.9 & 22.8 & 39.8 & 40.7 & 43.2 \\
\hline
\end{tabular}

*Turkish State Meteorological Service

The experiments in both years were evaluated in a randomized complete block design with three replications. Seeds of the genotypes were sown by hand on March 26 and 30, 2011 and 2012, respectively. Spacing between rows was $0.50 \mathrm{~m}$ and within rows spacing was $0.15 \mathrm{~m}$. The plot length was $5 \mathrm{~m}$ and each plot contained 5 rows.
All genotypes were fertilized with $8 \mathrm{~kg} \mathrm{da}^{-1}$ of $\mathrm{P}$ and $10 \mathrm{~kg}$ $\mathrm{da}^{-1}$ of N. Diammonium phosphate $(18 \% \mathrm{~N}, 46 \% \mathrm{P})$ and ammonium nitrate $(33 \% \mathrm{~N})$ were used as fertilizers. Weed control was performed by mechanical rotary tillage and manual weeding. Experiments were not irrigated. Genotypes were harvested on the first week of October in 
both years and only the three middle rows were harvested by hand. Agronomic and quality characteristics were determined in 10 randomly selected plants. The following observations were made: plant height $(\mathrm{cm})$, branches number (no plant ${ }^{-1}$ ), head number (no plant ${ }^{-1}$ ), head diameter (mm), 1000 seed weight $(\mathrm{g})$, harvest index $(\%)$, hull content $(\%)$, seed yield $\left(\mathrm{kg} \mathrm{da}^{-1}\right)$, oil content $(\%)$, oil yield $\left(\mathrm{kg} \mathrm{da}^{-1}\right)$, and fatty acid composition (\%).

The oil content and fatty acid composition were determined by Nuclear magnetic resonance (NMR, Bruker, the USA) and Gas chromatography (GC, Perkin Elmer Auto System XL, the USA), respectively. For the determination of oil content, the seeds of the genotypes were oven-dried at $35^{\circ} \mathrm{C}$ for 2 days in a ventilated oven till reaching constant moisture content and then the oil content of $2.5 \mathrm{~g}$ of seed was detected in NMR with 3 replications. To determine the fatty acid composition, the oil extracted with hexane/methanol $(4: 1, \mathrm{v} / \mathrm{v})$ from the seeds and oil was converted to its fatty acid methyl esters as described by Marquard (1987). The methyl esters of the fatty acids $(1.0 \mu \mathrm{l})$ were analyzed in a GC equipped with a flame ionizing detector (FID) and a fused silica capillary column (MNFFAP (50 m x $0.32 \mathrm{~mm}$ i.d.; film thickness = $0.25 \mu \mathrm{m})$ ). A GC analysis was performed as follows: the oven temperature was kept at $120^{\circ} \mathrm{C}$ for a min and programmed to $250^{\circ} \mathrm{C}$ at a rate of $6{ }^{\circ} \mathrm{C} \min ^{-1}$ and then constant at $240^{\circ} \mathrm{C}$ for $15 \mathrm{~min}$; total run time: $60 \mathrm{~min}$; injector temperature: $250^{\circ} \mathrm{C}$; detector $(70 \mathrm{eV})$ temperature: $260^{\circ} \mathrm{C}$; the flow rate for helium: $40 \mathrm{ml} \mathrm{min}^{-1}$; and split ratio: $1 / 20 \mathrm{ml} \mathrm{min}^{-1}$. Peak identification was conducted by comparing the relative retention times with those of a commercial standard mix of fatty acid methyl esters. The contents of palmitic (C16:0), stearic (C18:0), oleic (C18:1), and linoleic (C18:2) acids were determined in percentage. Samples were injected $1 \mu$ l. All data were analyzed using GLM producers of SAS (1999) and means were compared using Tukey's Multiple Range Test at the probability level of 0.05 .

\section{RESULTS AND DISCUSSION}

The results of the analysis of variance on the agronomic and quality characteristics examined in the research are given in Table 3 . According to the ANOVA results, the differences among the genotypes were found statistically significant $(\mathrm{P}<0.01)$ for all characteristics in 2011 and 2012. Likewise, according to the results of the combined ANOVA test, both cultivars and years and the year $\times$ genotype interaction were found to be significant. Since the differences between the years were significant in all characteristics, the years were given individually (Table 3).

Table 3. Results of the analysis of variance on the agronomic and quality characteristics.

\begin{tabular}{|c|c|c|c|c|c|c|c|c|c|c|c|c|}
\hline 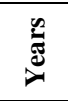 & $\begin{array}{l}\text { Sources of } \\
\text { variance }\end{array}$ & Df & $\begin{array}{c}\text { Plant } \\
\text { height }\end{array}$ & $\begin{array}{c}\text { Branches } \\
\text { number }\end{array}$ & $\begin{array}{c}\text { Head } \\
\text { number }\end{array}$ & $\begin{array}{c}\text { Head } \\
\text { diameter }\end{array}$ & $\begin{array}{c}1000 \\
\text { seed } \\
\text { weight }\end{array}$ & $\begin{array}{c}\text { Harvest } \\
\text { index }\end{array}$ & $\begin{array}{c}\text { Hull } \\
\text { content }\end{array}$ & $\begin{array}{l}\text { Seed } \\
\text { yield }\end{array}$ & $\begin{array}{c}\text { Oil } \\
\text { content }\end{array}$ & $\begin{array}{c}\text { Oil } \\
\text { yield }\end{array}$ \\
\hline \multirow{4}{*}{ 戸్ } & Block (B) & 2 & $4.7^{\mathrm{a}}$ & 0.4 & 0.6 & 0.1 & 5.3 & 3.2 & 1.4 & 40.2 & 1.3 & 8.1 \\
\hline & Genotype (G) & 38 & $254.7 * *$ & $11.4 * *$ & $29.0 * *$ & $7.4 * *$ & $56.9^{* *}$ & $80.1 * *$ & $92.4 * *$ & $5398.7 * *$ & $23.9 * *$ & $479.9 * *$ \\
\hline & Error & 76 & 7.7 & 0.4 & 1.0 & 0.4 & 3.3 & 2.7 & 2.4 & 137.9 & 0.9 & 13.0 \\
\hline & $\mathrm{CV}(\%)$ & & 5.3 & 8.8 & 8.7 & 3.0 & 5.1 & 8.4 & 3.4 & 13.5 & 3.3 & 14.2 \\
\hline \multirow{4}{*}{ తి } & $\mathrm{B}$ & 2 & 0.8 & 0.5 & 0.1 & 1.4 & 0.1 & 5.7 & 1.6 & 58.9 & 4.3 & 22.2 \\
\hline & G & 38 & $250.8^{* *}$ & $10.1^{* * *}$ & $51.6 * *$ & $9.8 * *$ & $55.5^{* *}$ & $120.3 * *$ & $32.2 * *$ & $5940.8^{* *}$ & $29.5 * *$ & $500.1 * *$ \\
\hline & Error & 76 & 8.8 & 0.7 & 2.4 & 1.0 & 3.8 & 4.5 & 2.4 & 208.3 & 2.1 & 17.9 \\
\hline & $\mathrm{CV}(\%)$ & & 5.3 & 9.0 & 9.8 & 4.5 & 5.2 & 9.8 & 3.3 & 13.5 & 5.0 & 14.2 \\
\hline \multirow{6}{*}{ 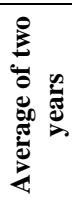 } & Year $(Y)$ & 1 & $91.4^{* *}$ & $51.9 * *$ & $867.6^{* * *}$ & $150.8 * *$ & $58.8^{* *}$ & $229.0 * *$ & $162.0^{* * *}$ & $23282.2 * *$ & $58.2 * *$ & $84.9 * *$ \\
\hline & $\mathrm{B}(\mathrm{Y})$ & 4 & 2.8 & 0.4 & 0.3 & 0.8 & 0.7 & 9.4 & 2.7 & 49.6 & 2.8 & 1.0 \\
\hline & G & 38 & $465.6^{* * *}$ & $19.5^{* * *}$ & $66.1 * *$ & $14.1^{* *}$ & $29.4^{* *}$ & $165.3^{* *}$ & $161.2^{* *}$ & $10714.9^{* *}$ & $48.9 * *$ & $59.2 * *$ \\
\hline & $Y \times G$ & 38 & $40.0^{* *}$ & $2.0^{* *}$ & $14.5^{* *}$ & $2.3^{* *}$ & $2.3^{* *}$ & $35.0 * *$ & $10.0 * *$ & $624.5 * *$ & $4.6^{* *}$ & $4.1^{* *}$ \\
\hline & Error & 152 & 8.1 & 0.5 & 1.7 & 0.7 & 3.6 & 3.6 & 2.4 & 173.1 & 1.5 & 15.5 \\
\hline & $\mathrm{CV}(\%)$ & & 5.3 & 8.9 & 9.5 & 3.9 & 5.1 & 9.2 & 3.3 & 13.6 & 4.2 & 14.2 \\
\hline
\end{tabular}

The highest plant height among the genotypes was observed in Yenice 5-38 $(84.1 \mathrm{~cm})$ in 2011. In 2012, Yenice 5-38 $(85.8 \mathrm{~cm})$ and Shufu $(82.4 \mathrm{~cm})$ had the highest plant height. The shortest plant height was detected in Enana $(38.2 \mathrm{~cm})$ and Gila $(42.6 \mathrm{~cm})$ in 2011 , Oleic Leed $(43.1 \mathrm{~cm})$ and NO 55-663 $(43.9 \mathrm{~cm})$ in 2012. Enana $(41.6 \mathrm{~cm})$ and Oleic Leed $(43.8 \mathrm{~cm})$ had the shortest plant height according to the average of both years (Table 4). Plant height has moderate to high heritability $(50.7-77.0 \%)$ (Ramachandram and Goud, 1981; Reddy et al., 2004; Parameshwar, 2009). Therefore it is also affected by the environmental conditions which were reported by different researchers (Camas et al., 2007; Beyyavas et al., 2011). Rainfall during growing period was higher in 2012 than 2011 which might explain observed differences for plant height between the years. 
Table 4. Agronomic and quality characteristics of domestic and foreign safflower genotypes

\begin{tabular}{|c|c|c|c|c|c|c|c|c|c|c|c|c|c|c|c|}
\hline \multirow{2}{*}{ Genotypes } & \multicolumn{3}{|c|}{ Plant height $(\mathbf{c m})$} & \multicolumn{3}{|c|}{ Branches number (no plant ${ }^{-1}$ ) } & \multicolumn{3}{|c|}{ Head number (no plant $\left.{ }^{-1}\right)$} & \multicolumn{3}{|c|}{ Head diameter (mm) } & \multicolumn{3}{|c|}{1000 seed weight $(\mathrm{g})$} \\
\hline & 2011 & 2012 & Mean & 2011 & 2012 & Mean & 2011 & 2012 & Mean & 2011 & 2012 & Mean & 2011 & 2012 & Mean \\
\hline Quiriego-88 & $52.3 \mathrm{f}-\mathrm{n}$ & $50.1 \mathrm{j}-\mathrm{p}$ & $51.2 \mathrm{~g}-\mathrm{o}$ & $9.4 \mathrm{~b}-\mathrm{f}$ & $9.0 \mathrm{c}-\mathrm{j}$ & $9.2 \mathrm{c}-\mathrm{g}$ & $20.5 \mathrm{a}$ & $24.0 \mathrm{ab}$ & $22.3 \mathrm{a}$ & $20.8 \mathrm{~d}-\mathrm{k}$ & $23.2 \mathrm{a}-\mathrm{i}$ & $22.0 \mathrm{c}-\mathrm{j}$ & $38.9 \mathrm{~b}-\mathrm{f}$ & $37.5 \mathrm{e}-\mathrm{k}$ & $38.2 \mathrm{~d}-\mathrm{i}$ \\
\hline Oker & $47.2 \mathrm{j}-\mathrm{p}$ & $49.4 j-p$ & $48.31-\mathrm{r}$ & $5.81-\mathrm{q}$ & $6.8 \mathrm{i}-\mathrm{m}$ & $6.3 \mathrm{~m}-\mathrm{p}$ & $10.2 \mathrm{~g}-\mathrm{n}$ & $16.1 \mathrm{~d}-\mathrm{j}$ & $13.2 \mathrm{f}-\mathrm{n}$ & $20.3 \mathrm{f}-\mathrm{m}$ & $21.3 \mathrm{f}-1$ & $20.8 \mathrm{~h}-\mathrm{m}$ & 22.61 & $26.8 \mathrm{~m}$ & $24.7 \mathrm{r}$ \\
\hline AC Sunset & $48.0 \mathrm{~h}-\mathrm{o}$ & $51.5 \mathrm{f}-\mathrm{p}$ & $49.7 \mathrm{j}-\mathrm{r}$ & $10.5 \mathrm{~b}-\mathrm{c}$ & $12.0 \mathrm{~b}$ & $11.3 \mathrm{~b}$ & $20.0 \mathrm{a}$ & $22.2 \mathrm{a}-\mathrm{c}$ & $21.1 \mathrm{ab}$ & $17.6 \mathrm{o}$ & $20.7 \mathrm{~h}-1$ & $19.21-\mathrm{o}$ & $35.1 \mathrm{~d}-\mathrm{k}$ & $36.9 \mathrm{e}-1$ & $36.0 \mathrm{~g}-\mathrm{o}$ \\
\hline Enana & $38.2 \mathrm{p}$ & $45.0 \mathrm{~m}-\mathrm{p}$ & $41.6 \mathrm{~s}$ & $7.4 \mathrm{f}-\mathrm{n}$ & $8.8 \mathrm{c}-\mathrm{k}$ & $8.1 \mathrm{e}-\mathrm{k}$ & $11.1 \mathrm{e}-\mathrm{m}$ & $14.1 \mathrm{~h}-\mathrm{m}$ & $12.6 \mathrm{~h}-\mathrm{p}$ & $18.41-\mathrm{o}$ & $20.9 \mathrm{~h}-1$ & $19.7 \mathrm{k}-\mathrm{o}$ & $32.5 \mathrm{~g}-\mathrm{k}$ & $36.5 \mathrm{e}-1$ & $34.5 \mathrm{~h}-\mathrm{q}$ \\
\hline Sahuaripa-88 & $45.2 \mathrm{j}-\mathrm{p}$ & $50.6 \mathrm{i}-\mathrm{p}$ & $47.9 \mathrm{~m}-\mathrm{s}$ & $7.3 \mathrm{f}-\mathrm{o}$ & $7.9 \mathrm{e}-\mathrm{m}$ & $7.6 \mathrm{~g}-\mathrm{m}$ & $12.5 \mathrm{~d}-\mathrm{I}$ & $16.8 \mathrm{~d}-\mathrm{i}$ & $14.7 \mathrm{~d}-\mathrm{k}$ & $21.6 \mathrm{c}-\mathrm{i}$ & $23.2 \mathrm{a}-\mathrm{i}$ & $22.4 \mathrm{~b}-\mathrm{h}$ & $42.6 \mathrm{~b}$ & 42.8 a-e & $42.7 \mathrm{bc}$ \\
\hline Montola-2000 & $62.0 \mathrm{c}-\mathrm{e}$ & $50.7 \mathrm{~h}-\mathrm{p}$ & $56.4 \mathrm{~d}-\mathrm{i}$ & $9.0 \mathrm{c}-\mathrm{g}$ & $9.1 \mathrm{c}-\mathrm{j}$ & $9.1 \mathrm{c}-\mathrm{g}$ & $16.1 \mathrm{~b}-\mathrm{c}$ & $21.0 \mathrm{~b}-\mathrm{d}$ & $18.5 \mathrm{bc}$ & $21.6 \mathrm{c}-\mathrm{i}$ & $24.0 \mathrm{a}-\mathrm{h}$ & $22.8 \mathrm{a}-\mathrm{g}$ & $36.6 \mathrm{c}-\mathrm{i}$ & 36.5 e-f & $36.6 \mathrm{e}-\mathrm{o}$ \\
\hline AC Stirling & $43.61-p$ & $50.6 \mathrm{~h}-\mathrm{p}$ & $47.1 \mathrm{n}-\mathrm{s}$ & $7.3 \mathrm{f}-\mathrm{o}$ & $8.5 \mathrm{~d}-1$ & $7.9 \mathrm{e}-1$ & $10.7 \mathrm{f}-\mathrm{m}$ & $16.5 \mathrm{~d}-\mathrm{i}$ & $13.6 \mathrm{f}-\mathrm{m}$ & $19.7 \mathrm{i}-\mathrm{n}$ & $21.9 \mathrm{c}-\mathrm{k}$ & $20.8 \mathrm{~h}-\mathrm{m}$ & $35.9 \mathrm{~d}-\mathrm{k}$ & $39.9 \mathrm{~b}-\mathrm{h}$ & $37.9 \mathrm{~d}-\mathrm{k}$ \\
\hline 4022 & $46.4 \mathrm{j}-\mathrm{p}$ & $60.2 \mathrm{~b}-\mathrm{h}$ & $53.3 \mathrm{f}-\mathrm{n}$ & $6.8 \mathrm{~h}-\mathrm{q}$ & $8.0 \mathrm{e}-\mathrm{m}$ & 7.4 h-n & $10.4 \mathrm{f}-\mathrm{n}$ & $20.7 \mathrm{~b}-\mathrm{e}$ & $15.5 \mathrm{~d}-\mathrm{h}$ & $22.4 \mathrm{a}-\mathrm{f}$ & $22.6 \mathrm{a}-\mathrm{j}$ & $22.5 \mathrm{~b}-\mathrm{h}$ & $30.3 \mathrm{k}$ & $35.1 \mathrm{~g}-1$ & $32.7 \mathrm{o}-\mathrm{q}$ \\
\hline PCA & $47.8 \mathrm{i}-\mathrm{o}$ & $53.8 \mathrm{~d}-\mathrm{n}$ & 50.8 h-p & $6.8 \mathrm{~h}-\mathrm{q}$ & $9.6 \mathrm{~b}-\mathrm{g}$ & $8.2 \mathrm{e}-\mathrm{j}$ & $9.2 \mathrm{i}-\mathrm{n}$ & $15.1 \mathrm{f}-\mathrm{k}$ & $12.1 \mathrm{i}-\mathrm{p}$ & $22.7 \mathrm{a}-\mathrm{e}$ & $24.8 \mathrm{a}-\mathrm{c}$ & $23.8 \mathrm{a}-\mathrm{c}$ & $35.7 \mathrm{~d}-\mathrm{k}$ & $41.4 \mathrm{a}-\mathrm{g}$ & $38.5 \mathrm{c}-\mathrm{h}$ \\
\hline Frio & $47.7 \mathrm{i}-\mathrm{o}$ & $53.9 \mathrm{~d}-\mathrm{n}$ & $50.8 \mathrm{~h}-\mathrm{q}$ & $8.5 \mathrm{c}-\mathrm{h}$ & $10.0 \mathrm{~b}-\mathrm{e}$ & $9.2 \mathrm{c}-\mathrm{f}$ & $10.6 \mathrm{f}-\mathrm{m}$ & $13.3 \mathrm{i}-\mathrm{n}$ & 12.0 k-p & $21.7 \mathrm{~b}-\mathrm{i}$ & $22.3 \mathrm{a}-\mathrm{j}$ & $22.0 \mathrm{c}-\mathrm{j}$ & $39.2 \mathrm{~b}-\mathrm{e}$ & $39.7 \mathrm{~b}-\mathrm{i}$ & $39.4 \mathrm{c}-\mathrm{g}$ \\
\hline Oleic Leed & 44.5 k-p & $43.1 \mathrm{p}$ & $43.8 \mathrm{r}-\mathrm{s}$ & $6.7 \mathrm{~h}-\mathrm{q}$ & $6.4 \mathrm{k}-\mathrm{m}$ & $6.6 \mathrm{k}-\mathrm{p}$ & 11.6d-1 & $12.8 \mathrm{i}-\mathrm{n}$ & $12.2 \mathrm{i}-\mathrm{p}$ & $22.8 \mathrm{a}-\mathrm{d}$ & $21.7 \mathrm{c}-1$ & $22.3 \mathrm{c}-\mathrm{i}$ & $35.2 \mathrm{~d}-\mathrm{k}$ & $37.4 \mathrm{e}-\mathrm{k}$ & $36.3 \mathrm{f}-\mathrm{o}$ \\
\hline Dincer 5-118 & $52.6 \mathrm{f}-\mathrm{m}$ & $57.0 \mathrm{c}-\mathrm{j}$ & $54.8 \mathrm{~d}-\mathrm{k}$ & $7.9 \mathrm{e}-1$ & $9.3 \mathrm{c}-\mathrm{h}$ & $8.6 \mathrm{~d}-\mathrm{i}$ & $14.7 \mathrm{~b}-\mathrm{d}$ & $18.8 \mathrm{c}-\mathrm{h}$ & $16.7 \mathrm{c}-\mathrm{e}$ & $23.8 \mathrm{a}$ & $25.4 \mathrm{a}-\mathrm{b}$ & $24.6 \mathrm{a}$ & $39.9 \mathrm{~b}-\mathrm{d}$ & $41.9 \mathrm{a}-\mathrm{f}$ & $40.9 \mathrm{~b}-\mathrm{e}$ \\
\hline Yenice 5-38 & $84.1 \mathrm{a}$ & $85.8 \mathrm{a}$ & $84.9 \mathrm{a}$ & $6.3 \mathrm{i}-\mathrm{q}$ & $7.3 \mathrm{f}-\mathrm{m}$ & $6.8 \mathrm{j}-\mathrm{p}$ & $10.7 \mathrm{f}-\mathrm{m}$ & $15.0 \mathrm{~g}-\mathrm{k}$ & $12.9 \mathrm{~g}-\mathrm{o}$ & $23.7 a-b$ & $24.7 \mathrm{a}-\mathrm{e}$ & $24.2 \mathrm{ab}$ & $37.2 \mathrm{~b}-\mathrm{h}$ & $37.7 \mathrm{~d}-\mathrm{j}$ & $37.5 \mathrm{~d}-1$ \\
\hline Remzibey-05 & 44.5 k-p & $46.8 \mathrm{f}-\mathrm{p}$ & $45.6 \mathrm{o}-\mathrm{s}$ & $6.2 \mathrm{j}-\mathrm{q}$ & $6.4 \mathrm{k}-\mathrm{m}$ & $6.3 \mathrm{~m}-\mathrm{p}$ & $13.6 \mathrm{c}-\mathrm{f}$ & $14.2 \mathrm{~h}-\mathrm{m}$ & $13.9 \mathrm{e}-\mathrm{m}$ & $21.1 \mathrm{c}-\mathrm{j}$ & $23.8 \mathrm{a}-\mathrm{h}$ & $22.5 \mathrm{~b}-\mathrm{h}$ & $37.0 \mathrm{~b}-\mathrm{h}$ & $37.9 \mathrm{~d}-\mathrm{j}$ & $37.4 \mathrm{~d}-\mathrm{m}$ \\
\hline Centennial & $57.1 \mathrm{c}-\mathrm{h}$ & $56.6 \mathrm{c}-\mathrm{k}$ & $56.8 \mathrm{c}-\mathrm{h}$ & $7.4 \mathrm{f}-\mathrm{n}$ & $7.8 \mathrm{e}-\mathrm{m}$ & $7.6 \mathrm{~g}-\mathrm{m}$ & $8.1 \mathrm{~m}-\mathrm{n}$ & $14.1 \mathrm{~h}-\mathrm{m}$ & $11.1 \mathrm{~m}-\mathrm{r}$ & $17.9 \mathrm{n}-\mathrm{o}$ & $18.8 \mathrm{k}-1$ & $18.4 \mathrm{o}$ & $30.91-\mathrm{k}$ & $31.8 \mathrm{j}-\mathrm{m}$ & $31.4 \mathrm{pq}$ \\
\hline S-517 & 51.9 g-n & $55.8 \mathrm{c}-1$ & $53.8 \mathrm{e}-\mathrm{m}$ & $9.3 \mathrm{~b}-\mathrm{f}$ & 9.6 b-g & $9.5 \mathrm{c}-\mathrm{e}$ & $12.0 \mathrm{~d}-\mathrm{j}$ & $18.7 \mathrm{c}-\mathrm{h}$ & 15.4 d-h & $21.0 \mathrm{c}-\mathrm{k}$ & $21.1 \mathrm{~g}-1$ & $21.0 \mathrm{~g}-1$ & $35.0 \mathrm{~d}-\mathrm{k}$ & $31.2 \mathrm{k}-\mathrm{m}$ & $33.1 \mathrm{n}-\mathrm{q}$ \\
\hline Lesaf & $46.1 \mathrm{j}-\mathrm{p}$ & 51.8 e-p & 48.9 k-r & $10.1 \mathrm{~b}-\mathrm{d}$ & $9.8 \mathrm{~b}-\mathrm{f}$ & $9.9 \mathrm{~b}-\mathrm{d}$ & $17.4 a-b$ & $26.3 \mathrm{a}$ & $21.9 \mathrm{a}$ & $19.7 \mathrm{i}-\mathrm{n}$ & 20.9 h-1 & $20.3 \mathrm{j}-\mathrm{n}$ & $30.3 \mathrm{f}-\mathrm{k}$ & $30.81-\mathrm{m}$ & $30.6 \mathrm{q}$ \\
\hline Saffire & $46.4 \mathrm{j}-\mathrm{p}$ & $54.3 \mathrm{~d}-\mathrm{m}$ & $50.3 \mathrm{i}-\mathrm{q}$ & $10.0 \mathrm{~b}-\mathrm{e}$ & $10.6 \mathrm{~b}-\mathrm{d}$ & $10.3 \mathrm{bc}$ & $13.2 \mathrm{c}-\mathrm{g}$ & $18.9 \mathrm{c}-\mathrm{h}$ & $16.1 \mathrm{c}-\mathrm{f}$ & $18.3 \mathrm{~m}-\mathrm{o}$ & $19.7 \mathrm{j}-1$ & $19.0 \mathrm{~m}-\mathrm{o}$ & $36.8 \mathrm{~b}-\mathrm{i}$ & $35.8 \mathrm{f}-1$ & $36.3 \mathrm{f}-\mathrm{o}$ \\
\hline Rehbein & $45.5 \mathrm{j}-\mathrm{p}$ & $51.1 \mathrm{~h}-\mathrm{p}$ & $48.3 \mathrm{k}-\mathrm{r}$ & $5.8 \mathrm{k}-\mathrm{q}$ & $7.1 \mathrm{~h}-\mathrm{m}$ & $6.51-p$ & $8.7 \mathrm{j}-\mathrm{n}$ & $15.4 \mathrm{f}-\mathrm{k}$ & $12.0 \mathrm{j}-\mathrm{p}$ & 19.0 k-o & $20.2 \mathrm{i}-1$ & 19.6 k-o & $33.0 \mathrm{f}-\mathrm{k}$ & $33.3 \mathrm{i}-1$ & $33.1 \mathrm{~m}-\mathrm{c}$ \\
\hline Finch & 44.3 k-p & $44.3 n-p$ & $44.3 \mathrm{q}-\mathrm{s}$ & $5.3 \mathrm{n}-\mathrm{q}$ & $5.7 \mathrm{~m}$ & $5.5 \mathrm{p}$ & $7.1 \mathrm{n}$ & $9.91-n$ & $8.5 \mathrm{q}-\mathrm{r}$ & $19.9 \mathrm{~h}-\mathrm{n}$ & $21.8 \mathrm{c}-1$ & $20.8 \mathrm{~h}-\mathrm{m}$ & $32.0 \mathrm{~h}-\mathrm{k}$ & $35.3 \mathrm{~g}-1$ & $33.7 \mathrm{k}-\mathrm{q}$ \\
\hline Leed & $58.3 \mathrm{c}-\mathrm{g}$ & $61.4 \mathrm{~b}-\mathrm{e}$ & $59.8 \mathrm{~b}-\mathrm{e}$ & $8.4 \mathrm{c}-\mathrm{i}^{\mathrm{T}}$ & $9.3 \mathrm{c}-\mathrm{h}$ & $8.8 \mathrm{c}-\mathrm{h}$ & $11.4 \mathrm{~d}-\mathrm{m}$ & 14.5 h-m & $12.9 \mathrm{~g}-\mathrm{o}$ & 22.5 a-e & $24.8 \mathrm{a}-\mathrm{d}$ & 23.7 a-d & $33.4 \mathrm{e}-\mathrm{k}$ & $37.4 \mathrm{e}-\mathrm{k}$ & $35.4 \mathrm{~g}-\mathrm{p}$ \\
\hline Arizona SC III & $46.0 \mathrm{j}-\mathrm{p}$ & $49.5 \mathrm{j}-\mathrm{p}$ & $47.7 \mathrm{~m}-\mathrm{s}$ & $6.8 \mathrm{~h}-\mathrm{q}$ & 7.7 e-m & $7.3 \mathrm{~h}-\mathrm{n}$ & $9.3 \mathrm{~h}-\mathrm{n}$ & $10.7 \mathrm{k}-\mathrm{n}$ & $10.0 \mathrm{o}-\mathrm{r}$ & $19.8 \mathrm{i}-\mathrm{n}$ & $21.4 \mathrm{e}-1$ & 20.6 h-n & $30.2 \mathrm{k}$ & $31.8 \mathrm{j}-\mathrm{m}$ & $31.0 \mathrm{q}$ \\
\hline NO 55-633 & $45.2 \mathrm{j}-\mathrm{p}$ & 43.9 o-p & $44.5 \mathrm{p}-\mathrm{s}$ & $6.6 \mathrm{~h}-\mathrm{q}$ & $6.31-\mathrm{m}$ & $6.41-p$ & $9.1 \mathrm{j}-\mathrm{n}$ & $13.1 \mathrm{i}-\mathrm{n}$ & $11.1 \mathrm{~m}-\mathrm{r}$ & $19.3 \mathrm{j}-\mathrm{o}$ & $21.5 \mathrm{~d}-1$ & 20.4 i-n & $38.1 \mathrm{~b}-\mathrm{g}$ & $44.7 \mathrm{a}-\mathrm{c}$ & $41.4 \mathrm{~b}-\mathrm{d}$ \\
\hline Hartman & $65.0 \mathrm{c}$ & $67.3 \mathrm{~b}$ & $66.2 \mathrm{~b}$ & $5.7 \mathrm{~m}-\mathrm{q}$ & $7.3 \mathrm{~g}-\mathrm{m}$ & $6.5 \mathrm{k}-\mathrm{p}$ & $10.8 \mathrm{f}-\mathrm{m}$ & 19.9 b-g & $15.3 \mathrm{~d}-\mathrm{h}$ & $22.0 \mathrm{a}-\mathrm{g}$ & $21.6 \mathrm{c}-1$ & $21.8 \mathrm{~d}-\mathrm{j}$ & $31.5 \mathrm{~h}-\mathrm{k}$ & $35.0 \mathrm{~g}-1$ & $33.31-q$ \\
\hline Ziyang & $43.4 \mathrm{~m}-\mathrm{p}$ & 47.4 k-p & $45.4 \mathrm{o}-\mathrm{s}$ & $4.9 \mathrm{p}-\mathrm{q}$ & $6.7 \mathrm{j}-\mathrm{m}$ & $5.8 \mathrm{n}-\mathrm{p}$ & $10.7 \mathrm{f}-\mathrm{m}$ & $11.9 \mathrm{i}-\mathrm{n}$ & $11.31-q$ & $20.6 \mathrm{e}-\mathrm{k}$ & $23.0 \mathrm{a}-\mathrm{i}$ & $21.8 \mathrm{~d}-\mathrm{j}$ & $49.1 \mathrm{a}$ & $46.6 \mathrm{a}$ & $47.8 \mathrm{a}$ \\
\hline Ole & $45.6 \mathrm{j}-\mathrm{p}$ & $46.0 \mathrm{~m}-\mathrm{p}$ & $45.8 \mathrm{o}-\mathrm{s}$ & $4.7 \mathrm{q}$ & $6.4 \mathrm{k}-\mathrm{m}$ & $5.6 \mathrm{op}$ & 8.4 k-n & $11.1 \mathrm{j}$-n & $9.8 \mathrm{pr}$ & $22.1 \mathrm{a}-\mathrm{g}$ & $24.4 \mathrm{a}-\mathrm{g}$ & $23.3 \mathrm{a}-\mathrm{e}$ & $34.2 \mathrm{~d}-\mathrm{k}$ & 37.0 e-f & $35.6 \mathrm{~g}-\mathrm{p}$ \\
\hline UC-1 & $48.2 \mathrm{~h}-\mathrm{o}$ & $49.9 \mathrm{j}-\mathrm{p}$ & $49.0 \mathrm{k}-\mathrm{r}$ & $5.2 \mathrm{o}-\mathrm{q}$ & $9.0 c-j$ & $7.1 \mathrm{i}-\mathrm{p}$ & 11.1 e-m & $15.8 \mathrm{e}-\mathrm{j}$ & $13.4 \mathrm{f}-\mathrm{m}$ & $21.9 \mathrm{a}-\mathrm{g}$ & $24.7 \mathrm{a}-\mathrm{e}$ & $23.3 \mathrm{a}-\mathrm{d}$ & $38.5 \mathrm{~b}-\mathrm{f}$ & $42.2 \mathrm{a}-\mathrm{f}$ & $40.3 \mathrm{~b}-\mathrm{e}$ \\
\hline US-10 & $52.7 \mathrm{f}-1$ & $56.9 \mathrm{c}-\mathrm{k}$ & $54.8 \mathrm{~d}-1$ & $6.9 \mathrm{~g}-\mathrm{p}$ & $7.4 \mathrm{f}-\mathrm{m}$ & $7.2 \mathrm{i}-\mathrm{o}$ & $11.1 \mathrm{e}-\mathrm{m}$ & $9.5 \mathrm{~m}-\mathrm{n}$ & $10.3 \mathrm{n}-\mathrm{r}$ & $21.5 \mathrm{c}-\mathrm{i}$ & $22.2 \mathrm{~b}-\mathrm{j}$ & $21.9 \mathrm{c}-\mathrm{j}$ & $39.4 \mathrm{~b}-\mathrm{e}$ & $42.4 \mathrm{a}-\mathrm{e}$ & $40.9 \mathrm{~b}-\mathrm{e}$ \\
\hline Rinconada & $62.3 \mathrm{c}-\mathrm{d}$ & $63.3 \mathrm{~b}-\mathrm{d}$ & $62.8 \mathrm{bc}$ & $14.1 \mathrm{a}$ & $14.7 \mathrm{a}$ & $14.4 \mathrm{a}$ & $16.6 \mathrm{~b}-\mathrm{c}$ & $14.6 \mathrm{~h}-1$ & $15.6 \mathrm{c}-\mathrm{g}$ & $18.61-0$ & $21.7 \mathrm{c}-1$ & $20.1 \mathrm{j}-\mathrm{o}$ & $36.3 \mathrm{~d}-\mathrm{j}$ & 40.1 b-h & $38.2 \mathrm{~d}-\mathrm{j}$ \\
\hline CH-353 & $53.7 \mathrm{~d}-\mathrm{j}$ & $57.5 \mathrm{c}-\mathrm{j}$ & $55.6 \mathrm{~d}-\mathrm{j}$ & $7.0 \mathrm{~g}-\mathrm{p}$ & $6.3 \mathrm{k}-\mathrm{m}$ & $6.6 \mathrm{j}-\mathrm{p}$ & $11.7 \mathrm{~d}-\mathrm{k}$ & $12.8 \mathrm{i}-\mathrm{n}$ & $12.3 \mathrm{i}-\mathrm{p}$ & $21.1 \mathrm{c}-\mathrm{j}$ & $21.5 \mathrm{e}-1$ & $21.3 \mathrm{f}-\mathrm{k}$ & $35.4 \mathrm{~d}-\mathrm{k}$ & $36.3 \mathrm{e}-1$ & $35.9 \mathrm{~g}-\mathrm{o}$ \\
\hline Sidwill & $56.8 \mathrm{c}-\mathrm{i}$ & $58.5 \mathrm{~b}-\mathrm{j}$ & $57.7 \mathrm{c}-\mathrm{g}$ & $11.5 \mathrm{~b}$ & $11.1 \mathrm{~b}-\mathrm{c}$ & $11.3 \mathrm{~b}$ & $10.7 \mathrm{f}-\mathrm{m}$ & $13.41-\mathrm{m}$ & $12.1 \mathrm{j}-\mathrm{p}$ & $21.2 \mathrm{c}-\mathrm{j}$ & $20.9 \mathrm{~h}-1$ & $21.1 \mathrm{~g}-1$ & $32.5 \mathrm{~g}-\mathrm{k}$ & $35.3 \mathrm{~g}-1$ & $33.9 \mathrm{j}-\mathrm{q}$ \\
\hline San Jose-89 & $59.5 \mathrm{c}-\mathrm{g}$ & $65.4 \mathrm{~b}-\mathrm{c}$ & $62.5 \mathrm{bc}$ & $6.6 \mathrm{~h}-\mathrm{q}$ & $9.2 \mathrm{c}-\mathrm{i}$ & $7.9 \mathrm{e}-1$ & $9.9 \mathrm{~g}-\mathrm{n}$ & $20.1 \mathrm{~b}-\mathrm{f}$ & $15.0 \mathrm{~d}-\mathrm{i}$ & $21.2 \mathrm{c}-\mathrm{j}$ & $24.5 \mathrm{a}-\mathrm{f}$ & $22.9 \mathrm{a}-\mathrm{g}$ & $35.2 \mathrm{~d}-\mathrm{k}$ & $39.6 \mathrm{~b}-\mathrm{i}$ & $37.4 \mathrm{~d}-\mathrm{n}$ \\
\hline Royal & $52.5 \mathrm{f}-\mathrm{m}$ & $63.4 \mathrm{~b}-\mathrm{d}$ & $58.0 \mathrm{c}-\mathrm{f}$ & $7.8 \mathrm{f}-\mathrm{m}$ & 7.7 e-m & $7.7 \mathrm{f}-\mathrm{m}$ & $10.7 \mathrm{f}-\mathrm{m}$ & $12.6 \mathrm{i}-\mathrm{n}$ & $11.61-p$ & $20.6 \mathrm{e}-\mathrm{k}$ & $25.6 \mathrm{a}$ & $23.1 \mathrm{a}-\mathrm{f}$ & $37.4 \mathrm{~b}-\mathrm{h}$ & $39.1 \mathrm{~b}-\mathrm{i}$ & $38.2 \mathrm{~d}-\mathrm{i}$ \\
\hline Gila & 42.6 o-p & $60.0 \mathrm{~b}-\mathrm{i}$ & $51.3 \mathrm{~g}-\mathrm{o}$ & $5.4 \mathrm{n}-\mathrm{q}$ & $10.2 \mathrm{~b}-\mathrm{e}$ & $7.8 \mathrm{f}-\mathrm{m}$ & $8.0 \mathrm{~m}-\mathrm{n}$ & $12.6 \mathrm{i}-\mathrm{n}$ & $10.3 \mathrm{n}-\mathrm{r}$ & $21.9 \mathrm{a}-\mathrm{h}$ & $24.5 \mathrm{a}-\mathrm{f}$ & $23.2 \mathrm{a}-\mathrm{e}$ & $36.1 \mathrm{~d}-\mathrm{k}$ & $37.4 \mathrm{e}-\mathrm{k}$ & $36.8 \mathrm{e}-\mathrm{o}$ \\
\hline Yuyao & $43.2 \mathrm{n}-\mathrm{p}$ & $60.6 \mathrm{~b}-\mathrm{g}$ & $51.9 \mathrm{f}-\mathrm{o}$ & $6.0 \mathrm{j}-\mathrm{q}$ & $7.4 \mathrm{f}-\mathrm{m}$ & $6.7 \mathrm{j}-\mathrm{p}$ & $11.5 \mathrm{~d}-1$ & $18.7 \mathrm{c}-\mathrm{h}$ & $15.1 \mathrm{~d}-\mathrm{i}$ & $20.2 \mathrm{~g}-\mathrm{m}$ & $22.6 \mathrm{a}-\mathrm{j}$ & $21.4 \mathrm{e}-\mathrm{k}$ & $42.3 \mathrm{~b}-\mathrm{c}$ & $45.5 \mathrm{a}-\mathrm{b}$ & $43.9 \mathrm{ab}$ \\
\hline Shufu & $74.8 \mathrm{~b}$ & $82.4 \mathrm{a}$ & $78.6 \mathrm{a}$ & $5.8 \mathrm{k}-\mathrm{q}$ & $7.1 \mathrm{~h}-\mathrm{m}$ & $6.51-p$ & $14.6 \mathrm{~b}-\mathrm{d}$ & $20.6 \mathrm{~b}-\mathrm{e}$ & $17.6 \mathrm{~cd}$ & 19.0 k-o & 18.61 & $18.8 \mathrm{n}-\mathrm{o}$ & $35.9 \mathrm{~d}-\mathrm{k}$ & $35.1 \mathrm{~g}-1$ & $35.5 \mathrm{~g}-\mathrm{p}$ \\
\hline Huaxian & $52.1 \mathrm{~g}-\mathrm{n}$ & $52.8 \mathrm{e}-\mathrm{o}$ & $52.5 \mathrm{f}-\mathrm{n}$ & $6.1 \mathrm{j}-\mathrm{q}$ & $6.81-\mathrm{m}$ & $6.41-p$ & $8.31-n$ & $8.3 \mathrm{n}$ & $8.3 \mathrm{r}$ & $20.4 \mathrm{f}-1$ & $21.2 \mathrm{f}-1$ & $20.8 \mathrm{~h}-\mathrm{m}$ & $34.3 \mathrm{~d}-\mathrm{k}$ & $33.9 \mathrm{~h}-1$ & $34.1 \mathrm{i}-\mathrm{q}$ \\
\hline FO-2 & $52.9 \mathrm{e}-\mathrm{k}$ & $56.1 \mathrm{c}-1$ & $54.5 \mathrm{e}-1$ & $7.9 \mathrm{~d}-\mathrm{k}$ & $6.7 \mathrm{j}-\mathrm{m}$ & $7.3 \mathrm{~h}-\mathrm{n}$ & $14.3 \mathrm{~b}-\mathrm{e}$ & $14.1 \mathrm{~h}-\mathrm{n}$ & $14.2 \mathrm{e}-1$ & $22.0 \mathrm{a}-\mathrm{g}$ & $24.6 \mathrm{a}-\mathrm{e}$ & $23.3 \mathrm{a}-\mathrm{e}$ & $39.2 \mathrm{~b}-\mathrm{e}$ & $38.7 \mathrm{c}-\mathrm{i}$ & $39.0 \mathrm{c}-\mathrm{g}$ \\
\hline Gifford & $61.4 c-p$ & $60.8 \mathrm{~b}-\mathrm{f}$ & $61.1 \mathrm{~b}-\mathrm{d}$ & $8.0 \mathrm{~d}-\mathrm{i}$ & $8.9 \mathrm{c}-\mathrm{j}$ & $8.5 \mathrm{~d}-\mathrm{i}$ & $12.6 \mathrm{~d}-\mathrm{h}$ & $11.1 \mathrm{j}-\mathrm{n}$ & 11.9 k-p & $22.9 \mathrm{a}-\mathrm{c}$ & $23.2 \mathrm{a}-\mathrm{i}$ & $23.1 \mathrm{a}-\mathrm{f}$ & $38.9 \mathrm{~b}-\mathrm{f}$ & $44.1 \mathrm{a}-\mathrm{d}$ & $41.5 \mathrm{~b}-\mathrm{d}$ \\
\hline
\end{tabular}


The number of branches per plant ranged from 4.7 to 14.7 in the study. While the highest number of branches was observed in Rinconado (14.1 in 2011 and 14.7 in 2012) and for the both years, the lowest number of branches was detected in Ole (4.7) in 2011. For combined years, there was no difference between Ole and Finch (Table 4). Weiss (2000) emphasized that 6 to 8 branches should be available in the plant for economic safflower production. However, some researchers reported that the number of branches might increase up to 50 in safflower (Deokar and Patil, 1980; Reddy et al., 2004; Omidi et al., 2009). Our results were found within the limits reported by these researchers. Singh et al. (2008) reported that the additive genes had an effect on the inheritance of the number of branches. Our results ranged from 5.5 to 14.4 according to the average of both years, which suggest that there is genetic variation present for the branch number among the studied genotypes.

In the study, Quiriego-88 and AC Sunset were found to have the largest number of heads per plant in 2011, in 2012, and according to the average of both years. Besides, Lesaf (26.3 and 21.9) and AC Sunset (22.2 and 21.1) in 2012 and according to the average of both years also gave high values for number of heads per plant. Finch and Huaxian had the fewest number of heads per plant for 2011, 2012 and combined years. The number of heads for Dincer 5-118, Yenice 5-38 and Remzibey-05 were determined as 16.7, 12.9, and 13.9 according to the average of both years, respectively (Table 4). Even though the number of heads is a characteristic which may vary by the environmental and cultural conditions (particularly by the sowing density) in safflower, it is one of the selection criteria with a direct effect on the high seed yield in early generations in a breeding program (Knowles, 1982). It was also reported that there is a high heritability for the number of heads in safflower and that the variation likely to occur is due to the environmental factors (Pahlavani et al., 2007; Parameshwar, 2009; Safavi et al., 2011). Weiss (2000) stated that although the number of heads ranged from 5 to 50 in safflower, 14 to 16 heads which displayed good development were enough for an economic yield. The variation determined in terms of the number of heads in this study is between the values stated in the other studies performed on the subject (Reddy et al., 2004; Safavi et al., 2011).

In both study years, head diameter significantly varied by year and genotype. Dincer 5-118, Yenice 5-38, Gifford, Oleic Leed, PCA, Leed, 4022, Ole, FO-2, Hartman, UC-1 and Gila in the first year of the study and Royal, Dincer 5-118, PCA, Leed, UC-1, Yenice 5-38, FO2, San Jose-89, Gila, Ole, Montola 2000, Gifford, Sahuaripa-88, Quiriego-88, Ziyang, Yuyao, 4022, Frio and Remzibey-05 in the second year statistically belonged to the same group. According to the average of both years, the maximum head diameter was recorded in Dincer 5118, Yenice 5-38, PCA, Leed, UC-1, FO-2, Ole, Gila, Royal and Gifford, San Jose-89, and Montola 2000. AC Sunset was detected to be the genotype with the minimum head diameter in 2011, Shufu in 2012, and Centennial according to the average of both years (Table 4). In the second growing season, bigger heads were obtained because of a higher total amount of rainfall and its best monthly distribution. It was reported that the head diameter could range from 10.0 to $35.0 \mathrm{~mm}$ in safflower (Weiss, 2000; Kizil et al., 2008) and that head diameter might vary by location, genotype, and climate (Safavi et al., 2011).

Another essential selection criterion that determines seed yield in safflower is the 1000 seed weight. The 1000 seed weight of the genotypes was in the range of 22.6$49.1 \mathrm{~g}$ in 2011, in the range of 26.8-46.6 $\mathrm{g}$ in 2012, and among 24.7-47.8 $\mathrm{g}$ according to the average of both years. In the study, the highest 1000 seed weight was determined in Ziyang, followed by Yuyao (45.5 g), NO 55-663 (44.7 g), Gifford (44.1 g), Sahuaripa-88 (42.8 g), US-10 (42.4 g), UC-1 (42.2 g), Dincer 5-118 (41.9 g), and PCA (41.4 g) in 2012 (Table 4). Ziyang and Yuyao had the highest values for 1000 seed weight and Oker had the lowest value for 1000 seed weight for combined years. The 1000 seed weight demonstrated that variations existed among the genotypes. Weiss (2000) reported that the high 1000 seed weight up to $50 \mathrm{~g}$ in safflower might increase the seed and/or oil yield. Detection of higher 1000 seed weights in 2012 might have contributed to high seed and oil yields observed in the second year of the study. Nevertheless, no genotype with a 1000 seed weight greater than $50 \mathrm{~g}$ was detected in either experimental year. The reported results were within the previously reported limits (Reddy et al., 2004; Alizadeh, 2005; Camas and Esendal, 2006; Safavi et al., 2011).

The harvest indexes of the genotypes ranged from 8.5 to $31.5 \%$ (Shufu-Gifford) and from 4.1 to $30.8 \%$ (OkerRemzibey-05) in 2011 and 2012, respectively. The highest harvest index according to the average of both years was recorded from Gifford and Remzibey-05, followed by US10, Dincer 5-118, San Jose-89, Gila, and Ziyang (Table $5)$. The effects of environmental factors are important on the realized harvest index in safflower. The high precipitation $(126.6 \mathrm{~mm})$ in May and June, bolting and budding periods of the plants, in 2012 should have contributed higher value of harvest index by directly increasing head diameter and 1000 seed weights of the genotypes. The results obtained in our study were found to be in agreement with the previous studies (Lakshmi Prayaga et al., 2003; Eslam, 2010). However, higher harvest index (>35\%) value was reported among different safflower genotypes (Parameshwar, 2009; Emami et al., 2011). 
Table 5. Agronomic and quality characteristics of domestic and foreign safflower genotypes

\begin{tabular}{|c|c|c|c|c|c|c|c|c|c|c|c|c|c|c|c|}
\hline \multirow{2}{*}{ Genotypes } & \multicolumn{3}{|c|}{ Harvest index (\%) } & \multicolumn{3}{|c|}{ Hull content (\%) } & \multicolumn{3}{|c|}{ Seed yield $\left(\mathrm{kg} \mathrm{da}^{-1}\right)$} & \multicolumn{3}{|c|}{ Oil content $(\%)$} & \multicolumn{3}{|c|}{ Oil yield $\left(\mathrm{kg} \mathrm{da}^{-1}\right)$} \\
\hline & 2011 & 2012 & Mean & 2011 & 2012 & Mean & 2011 & 2012 & Mean & 2011 & 2012 & Mean & 2011 & 2012 & Mean \\
\hline Quiriego-88 & $22.6 \mathrm{c}-\mathrm{g}$ & $21.2 \mathrm{c}-\mathrm{i}$ & $21.9 \mathrm{e}-\mathrm{k}$ & $47.7 \mathrm{~d}-1$ & $49.0 \mathrm{~d}-\mathrm{k}$ & $48.3 \mathrm{~d}-\mathrm{h}$ & $114.1 \mathrm{~d}-\mathrm{g}$ & $116.6 \mathrm{~d}-\mathrm{k}$ & $115.3 \mathrm{~d}-\mathrm{j}$ & $29.5 \mathrm{e}-\mathrm{k}$ & $26.4 \mathrm{~g}-\mathrm{m}$ & $28.0 \mathrm{~g}-\mathrm{o}$ & $33.6 \mathrm{~d}-\mathrm{f}$ & $30.8 \mathrm{f}-\mathrm{m}$ & $32.2 \mathrm{e}-\mathrm{j}$ \\
\hline Oker & $17.7 \mathrm{~g}-1$ & 4.11 & $10.9 \mathrm{pq}$ & $35.1 \mathrm{o}$ & $46.5 \mathrm{~h}-\mathrm{m}$ & 40.8 n-o & $29.0 \mathrm{p}-\mathrm{r}$ & 19.5 o-p & $24.2 \mathrm{t}-\mathrm{u}$ & 32.6 a-e & $28.4 \mathrm{c}-\mathrm{k}$ & $30.5 \mathrm{c}-\mathrm{g}$ & $9.5 \mathrm{~m}-\mathrm{o}$ & $5.5 \mathrm{p}-\mathrm{q}$ & 7.5 q-r \\
\hline AC Sunset & $16.7 \mathrm{~h}-1$ & $17.6 \mathrm{~g}-\mathrm{j}$ & $17.2 \mathrm{~m}-\mathrm{o}$ & $50.7 \mathrm{~b}-\mathrm{f}$ & $52.2 \mathrm{a}-\mathrm{f}$ & $51.5 \mathrm{a}-\mathrm{d}$ & $83.1 \mathrm{f}-\mathrm{m}$ & $112.2 \mathrm{~d}-1$ & $97.7 \mathrm{~h}-\mathrm{m}$ & $25.0 \mathrm{~m}-\mathrm{p}$ & $26.1 \mathrm{~h}-\mathrm{m}$ & $25.6 \mathrm{o}-\mathrm{q}$ & 20.8 h-m & $29.2 \mathrm{f}-\mathrm{n}$ & $25.0 \mathrm{i}-\mathrm{n}$ \\
\hline Sahuaripa-88 & $19.6 e-j$ & $21.9 \mathrm{c}-\mathrm{i}$ & $20.7 \mathrm{~g}-\mathrm{m}$ & $45.5 \mathrm{~g}-\mathrm{m}$ & $47.7 \mathrm{f}-1$ & $46.6 \mathrm{~g}-\mathrm{k}$ & 109.4 e-h & $123.5 \mathrm{~d}-\mathrm{i}$ & $116.4 \mathrm{~d}-\mathrm{i}$ & $31.4 \mathrm{~b}-\mathrm{g}$ & $30.1 \mathrm{a}-\mathrm{i}$ & $30.7 \mathrm{~b}-\mathrm{g}$ & $34.4 \mathrm{~d}-\mathrm{f}$ & $37.2 \mathrm{c}-\mathrm{g}$ & $35.8 \mathrm{~d}-\mathrm{f}$ \\
\hline Montola-2000 & $18.5 \mathrm{~g}-\mathrm{k}$ & $20.7 \mathrm{~d}-\mathrm{j}$ & 19.6 g-n & $43.2 \mathrm{j}-\mathrm{n}$ & $42.0 \mathrm{~m}-\mathrm{n}$ & $42.6 \mathrm{~m}-\mathrm{n}$ & $157.0 \mathrm{~b}-\mathrm{c}$ & $156.9 \mathrm{~b}-\mathrm{e}$ & $157.0 \mathrm{bc}$ & $31.0 \mathrm{~b}-\mathrm{h}$ & $29.5 b-j$ & $30.3 c-h$ & $48.7 \mathrm{~b}$ & $46.4 \mathrm{~b}-\mathrm{e}$ & $47.5 \mathrm{~b}-\mathrm{c}$ \\
\hline AC Stirling & $19.1 \mathrm{f}-\mathrm{k}$ & $19.3 e-j$ & $19.2 \mathrm{~h}-\mathrm{n}$ & $48.2 c-j$ & $53.0 \mathrm{a}-\mathrm{e}$ & $50.6 \mathrm{c}-\mathrm{f}$ & $53.51-\mathrm{r}$ & $85.1 \mathrm{~g}-\mathrm{n}$ & $69.3 \mathrm{~m}-\mathrm{s}$ & 28.2 h-m & $24.9 \mathrm{j}-\mathrm{m}$ & $26.6 \mathrm{~m}-\mathrm{p}$ & $15.0 \mathrm{k}-\mathrm{o}$ & $21.2 \mathrm{i}-\mathrm{o}$ & $18.1 \mathrm{~m}-\mathrm{p}$ \\
\hline 4022 & $17.7 \mathrm{~g}-1$ & $20.2 \mathrm{e}-\mathrm{j}$ & $19.0 \mathrm{j}-\mathrm{n}$ & $50.8 \mathrm{~b}-\mathrm{f}$ & $51.2 \mathrm{~b}-\mathrm{h}$ & $51.0 \mathrm{~b}-\mathrm{e}$ & $68.6 \mathrm{j}-\mathrm{o}$ & $130.5 \mathrm{~d}-\mathrm{h}$ & $99.6 \mathrm{~g}-1$ & $26.31-0$ & $26.5 \mathrm{f}-\mathrm{m}$ & $26.4 \mathrm{~m}-\mathrm{p}$ & $18.1 \mathrm{i}-\mathrm{n}$ & $34.8 \mathrm{~d}-\mathrm{i}$ & $26.4 \mathrm{~g}-\mathrm{m}$ \\
\hline PCA & $13.9 \mathrm{k}-\mathrm{n}$ & $23.3 \mathrm{c}-\mathrm{i}$ & $18.6 \mathrm{k}-\mathrm{n}$ & $43.2 \mathrm{j}-\mathrm{n}$ & $47.0 \mathrm{~g}-\mathrm{m}$ & 45.1 h-m & $74.6 \mathrm{~h}-\mathrm{n}$ & $116.7 \mathrm{~d}-\mathrm{k}$ & $95.7 \mathrm{~h}-\mathrm{n}$ & $28.8 \mathrm{~g}-1$ & $30.8 \mathrm{a}-\mathrm{h}$ & $29.8 \mathrm{c}-\mathrm{g}$ & $21.5 \mathrm{~g}-1$ & $35.9 \mathrm{c}-\mathrm{h}$ & $28.7 \mathrm{f}-\mathrm{k}$ \\
\hline Oleic & $22.5 \mathrm{c}-\mathrm{g}$ & $24.2 \mathrm{a}-\mathrm{h}$ & $23.3 \mathrm{c}-\mathrm{i}$ & $42.71-n$ & $46.1 \mathrm{~h}-\mathrm{m}$ & $44.4 \mathrm{j}-\mathrm{m}$ & $76.7 \mathrm{~g}-\mathrm{n}$ & $80.4 \mathrm{i}-\mathrm{n}$ & $78.61-\mathrm{r}$ & $34.0 \mathrm{a}-\mathrm{b}$ & $33.5 \mathrm{ab}$ & $33.8 \mathrm{a}$ & $26.1 \mathrm{e}-\mathrm{k}$ & $26.8 \mathrm{~g}-\mathrm{n}$ & $26.4 \mathrm{~g}-\mathrm{m}$ \\
\hline Dincer 5-118 & $28.8 \mathrm{a}-\mathrm{b}$ & $27.7 \mathrm{a}-\mathrm{d}$ & $28.2 \mathrm{ab}$ & $51.8 \mathrm{a}-\mathrm{e}$ & $49.6 \mathrm{~d}-\mathrm{j}$ & 50.7 b-f & $165.7 \mathrm{~b}$ & $183.3 \mathrm{a}-\mathrm{c}$ & $174.5 \mathrm{~b}$ & $28.8 \mathrm{~g}-1$ & $26.4 \mathrm{~g}-\mathrm{m}$ & $27.6 \mathrm{~h}-\mathrm{o}$ & $47.7 \mathrm{~b}-\mathrm{c}$ & $48.3 \mathrm{~b}-\mathrm{d}$ & $48.0 \mathrm{~b}-\mathrm{c}$ \\
\hline Yenice 5-38 & $18.2 \mathrm{~g}-1$ & $16.9 \mathrm{i}-\mathrm{j}$ & $17.51-\mathrm{o}$ & $52.3 \mathrm{a}-\mathrm{d}$ & $56.1 \mathrm{ab}$ & $54.2 \mathrm{a}-\mathrm{b}$ & $130.5 \mathrm{~b}-\mathrm{e}$ & $131.4 \mathrm{~d}-\mathrm{g}$ & $130.9 \mathrm{c}-\mathrm{f}$ & $24.7 \mathrm{n}-\mathrm{p}$ & $24.2 \mathrm{k}-\mathrm{m}$ & $24.4 \mathrm{q}-\mathrm{r}$ & $32.2 \mathrm{~d}-\mathrm{h}$ & $31.8 \mathrm{f}-\mathrm{k}$ & $32.0 \mathrm{e}-\mathrm{j}$ \\
\hline Remzibey-05 & $28.7 a-b$ & $30.8 \mathrm{a}$ & $29.7 \mathrm{a}$ & 49.4 b-h & $51.9 \mathrm{a}-\mathrm{g}$ & 50.7 b-f & 107.7 e-i & $116.7 \mathrm{~d}-\mathrm{k}$ & $112.2 \mathrm{e}-\mathrm{j}$ & $30.5 c-i$ & $28.3 \mathrm{~d}-\mathrm{k}$ & $29.4 \mathrm{~d}-1$ & $32.9 \mathrm{~d}-\mathrm{g}$ & $33.0 e-j$ & $32.9 \mathrm{e}-\mathrm{i}$ \\
\hline Centennial & $19.0 \mathrm{f}-\mathrm{k}$ & $8.3 \mathrm{k}-1$ & 13.7 op & 38.7 n-o & $38.9 \mathrm{n}$ & $38.8 \mathrm{o}$ & $50.1 \mathrm{~m}-\mathrm{r}$ & $50.4 \mathrm{n}-\mathrm{p}$ & $50.3 \mathrm{r}-\mathrm{t}$ & $35.0 \mathrm{a}$ & $32.6 \mathrm{a}-\mathrm{d}$ & $33.8 \mathrm{a}$ & $17.6 \mathrm{i}-\mathrm{o}$ & $16.4 \mathrm{n}-\mathrm{q}$ & $17.0 \mathrm{n}-\mathrm{p}$ \\
\hline S-517 & $22.2 \mathrm{c}-\mathrm{h}$ & $21.7 \mathrm{c}-\mathrm{i}$ & $21.9 \mathrm{e}-\mathrm{k}$ & 42.9 k-n & $45.1 \mathrm{j}-\mathrm{m}$ & 44.0 k-n & $65.5 \mathrm{j}-\mathrm{p}$ & $51.7 \mathrm{n}-\mathrm{p}$ & $58.6 \mathrm{o}-\mathrm{s}$ & $30.0 \mathrm{~d}-\mathrm{j}$ & $33.6 \mathrm{ab}$ & $31.8 \mathrm{a}-\mathrm{d}$ & $19.6 \mathrm{i}-\mathrm{n}$ & $17.4 \mathrm{l-q}$ & $18.51-\mathrm{p}$ \\
\hline Lesaf & $20.0 \mathrm{~d}-\mathrm{i}$ & $25.5 \mathrm{a}-\mathrm{f}$ & $22.7 \mathrm{c}-\mathrm{k}$ & $46.0 \mathrm{f}-\mathrm{m}$ & $47.2 \mathrm{f}-\mathrm{m}$ & $46.6 \mathrm{~g}-\mathrm{k}$ & $84.0 \mathrm{f}-\mathrm{m}$ & $126.0 \mathrm{~d}-\mathrm{i}$ & $105.0 \mathrm{f}-1$ & $30.6 \mathrm{c}-\mathrm{i}$ & 26.8 e-m & 28.7 e-m & $25.7 \mathrm{e}-\mathrm{k}$ & $33.7 \mathrm{e}-\mathrm{i}$ & $29.7 \mathrm{e}-\mathrm{k}$ \\
\hline Saffire & $19.0 \mathrm{f}-\mathrm{k}$ & $25.0 \mathrm{a}-\mathrm{f}$ & $22.0 \mathrm{e}-\mathrm{k}$ & $52.1 \mathrm{a}-\mathrm{d}$ & $49.0 \mathrm{~d}-\mathrm{k}$ & $50.5 \mathrm{c}-\mathrm{f}$ & 33.3 o-r & $74.3 \mathrm{j}-\mathrm{n}$ & $53.8 \mathrm{q}-\mathrm{t}$ & $28.0 \mathrm{~h}-\mathrm{m}$ & $24.9 \mathrm{j}-\mathrm{m}$ & $26.4 \mathrm{~m}-\mathrm{p}$ & $9.3 \mathrm{~m}-\mathrm{o}$ & $18.5 \mathrm{k}-\mathrm{q}$ & 13.9 p-r \\
\hline Rehbein & $13.9 \mathrm{k}-\mathrm{n}$ & $23.6 \mathrm{~b}-\mathrm{i}$ & $18.7 \mathrm{k}-\mathrm{n}$ & $44.9 \mathrm{~h}-1$ & $47.0 \mathrm{~g}-\mathrm{m}$ & 45.9 h-m & $62.7 \mathrm{k}-\mathrm{q}$ & $61.1 \mathrm{~m}-\mathrm{o}$ & $61.9 \mathrm{o}-\mathrm{s}$ & $29.2 \mathrm{f}-1$ & $27.7 \mathrm{e}-\mathrm{k}$ & $28.4 \mathrm{f}-\mathrm{n}$ & $18.2 \mathrm{i}-\mathrm{n}$ & $16.9 \mathrm{~m}-\mathrm{q}$ & $17.6 \mathrm{~m}-\mathrm{p}$ \\
\hline Finch & $19.6 e-j$ & $25.2 \mathrm{a}-\mathrm{f}$ & $22.4 \mathrm{~d}-\mathrm{k}$ & $43.9 \mathrm{i}-1$ & 43.7 1-n & 43.8 k-n & $48.7 \mathrm{~m}-\mathrm{r}$ & 83.7 h-n & $66.2 \mathrm{n}-\mathrm{s}$ & $33.5 \mathrm{a}-\mathrm{c}$ & $33.1 \mathrm{a}-\mathrm{c}$ & $33.3 \mathrm{ab}$ & $16.4 \mathrm{j}$-o & $27.7 \mathrm{f}-\mathrm{n}$ & $22.0 \mathrm{k}-\mathrm{p}$ \\
\hline Leed & $14.3 \mathrm{j}-\mathrm{m}$ & $22.6 c-i$ & 18.4 k-n & $43.7 \mathrm{i}-\mathrm{n}$ & $45.3 \mathrm{i}-\mathrm{m}$ & $44.51-\mathrm{m}$ & 83.0 f-m & $90.3 \mathrm{f}-\mathrm{n}$ & $86.7 \mathrm{i}-\mathrm{o}$ & $31.6 \mathrm{~b}-\mathrm{g}$ & $31.2 \mathrm{a}-\mathrm{f}$ & $31.4 \mathrm{a}-\mathrm{e}$ & $26.4 \mathrm{e}-\mathrm{k}$ & $28.2 \mathrm{f}-\mathrm{n}$ & $27.3 \mathrm{f}-1$ \\
\hline Hartman & $22.6 \mathrm{c}-\mathrm{g}$ & $22.3 \mathrm{~d}-\mathrm{i}$ & $22.4 \mathrm{~d}-\mathrm{k}$ & $46.6 \mathrm{e}-1$ & $48.1 \mathrm{e}-1$ & $47.4 \mathrm{f}-\mathrm{k}$ & 75.4 g-n & $112.1 \mathrm{e}-\mathrm{f}$ & $93.7 \mathrm{~h}-\mathrm{n}$ & $27.3 \mathrm{j}-\mathrm{n}$ & $26.1 \mathrm{i}-\mathrm{m}$ & $26.71-p$ & 20.6 h-m & $29.2 \mathrm{f}-\mathrm{n}$ & $24.9 \mathrm{i}-\mathrm{n}$ \\
\hline Ziyang & $24.4 b-f$ & $27.7 \mathrm{a}-\mathrm{c}$ & $26.1 \mathrm{a}-\mathrm{e}$ & $561 \mathrm{a}$ & $53.7 \mathrm{a}-\mathrm{d}$ & $54.9 \mathrm{a}$ & $117.3 \mathrm{~d}-\mathrm{f}$ & $118.0 \mathrm{~d}-\mathrm{j}$ & 117.7 d-h & $24.1 \mathrm{o}-\mathrm{p}$ & $22.91-\mathrm{m}$ & $23.5 \mathrm{q}-\mathrm{r}$ & $28.2 \mathrm{e}-\mathrm{j}$ & $27.1 \mathrm{~g}-\mathrm{n}$ & $27.7 \mathrm{f}-\mathrm{k}$ \\
\hline Ole & $22.0 \mathrm{c}-\mathrm{h}$ & $21.6 \mathrm{c}-\mathrm{i}$ & $21.8 \mathrm{e}-1$ & $46.5 \mathrm{f}-1$ & $50.3 \mathrm{c}-1$ & 48.4d-h & $104.0 \mathrm{e}-\mathrm{j}$ & $113.4 \mathrm{~d}-\mathrm{k}$ & $108.7 \mathrm{f}-\mathrm{k}$ & $33.0 \mathrm{a}-\mathrm{d}$ & $31.5 \mathrm{a}-\mathrm{e}$ & $32.2 \mathrm{a}-\mathrm{c}$ & $34.3 \mathrm{~d}-\mathrm{f}$ & $35.6 \mathrm{c}-\mathrm{h}$ & $35.0 \mathrm{~d}-\mathrm{g}$ \\
\hline UC-1 & $24.8 \mathrm{~b}-\mathrm{e}$ & $24.6 \mathrm{a}-\mathrm{g}$ & 24.7 b-f & $47.7 \mathrm{~d}-1$ & $47.9 \mathrm{e}-1$ & $47.8 \mathrm{e}-\mathrm{j}$ & $208.8 \mathrm{a}$ & $223.0 \mathrm{a}$ & $215.9 \mathrm{a}$ & $30.5 \mathrm{c}-\mathrm{i}$ & $29.8 \mathrm{a}-\mathrm{i}$ & $30.2 \mathrm{c}-\mathrm{i}$ & $63.7 \mathrm{a}$ & $66.6 \mathrm{a}$ & $65.1 \mathrm{a}$ \\
\hline US-10 & $28.8 \mathrm{a}-\mathrm{b}$ & $30.4 \mathrm{a}-\mathrm{b}$ & $29.6 \mathrm{a}$ & $46.8 \mathrm{e}-1$ & $47.9 \mathrm{e}-1$ & $47.4 \mathrm{f}-\mathrm{k}$ & $119.2 \mathrm{c}-\mathrm{f}$ & $159.8 \mathrm{~b}-\mathrm{d}$ & $139.5 \mathrm{c}-\mathrm{e}$ & $30.0 \mathrm{~d}-\mathrm{j}$ & $30.8 \mathrm{a}-\mathrm{h}$ & $30.4 \mathrm{c}-\mathrm{g}$ & $35.9 \mathrm{c}-\mathrm{e}$ & $49.2 \mathrm{bc}$ & $42.6 \mathrm{c}-\mathrm{d}$ \\
\hline Rinconada & $18.1 \mathrm{~g}-1$ & $18.7 \mathrm{f}-\mathrm{j}$ & 18.4 k-n & $43.4 \mathrm{i}-\mathrm{n}$ & $46.0 \mathrm{i}-\mathrm{m}$ & $44.7 \mathrm{i}-\mathrm{m}$ & $70.7 \mathrm{~h}-\mathrm{o}$ & $100.6 \mathrm{f}-\mathrm{m}$ & 85.7 j-p & $29.5 \mathrm{e}-\mathrm{k}$ & $30.8 \mathrm{a}-\mathrm{h}$ & $30.2 \mathrm{c}-\mathrm{i}$ & $20.9 \mathrm{~h}-\mathrm{m}$ & $31.0 \mathrm{f}-1$ & $25.9 \mathrm{~h}-\mathrm{n}$ \\
\hline CH-353 & $18.3 \mathrm{~g}-1$ & $22.8 c-i$ & $20.6 \mathrm{~g}-\mathrm{n}$ & $45.2 \mathrm{~h}-1$ & $48.5 \mathrm{e}-1$ & $46.9 \mathrm{~g}-\mathrm{k}$ & $70.2 \mathrm{i}-\mathrm{o}$ & $91.2 \mathrm{f}-\mathrm{n}$ & $80.7 \mathrm{k}-\mathrm{q}$ & $29.4 \mathrm{f}-1$ & $29.6 \mathrm{a}-\mathrm{j}$ & $29.5 \mathrm{c}-\mathrm{k}$ & 20.6 h-m & $27.0 \mathrm{~g}-\mathrm{n}$ & $23.8 \mathrm{j}-\mathrm{o}$ \\
\hline Sidwill & $15.6 \mathrm{i}-1$ & $22.6 c-i$ & $19.1 \mathrm{i}-\mathrm{n}$ & $46.8 \mathrm{e}-1$ & $48.8 \mathrm{~d}-1$ & $47.8 \mathrm{e}-\mathrm{j}$ & $71.2 \mathrm{~h}-\mathrm{o}$ & $81.6 \mathrm{i}-\mathrm{n}$ & $76.41-\mathrm{r}$ & $27.5 \mathrm{i}-\mathrm{n}$ & $27.1 \mathrm{e}-1$ & $27.3 \mathrm{j}-\mathrm{o}$ & $19.7 \mathrm{i}-\mathrm{n}$ & $22.2 \mathrm{~h}-\mathrm{o}$ & $21.0 \mathrm{k}-\mathrm{p}$ \\
\hline San Jose-89 & $26.1 \mathrm{a}-\mathrm{c}$ & $27.8 \mathrm{a}-\mathrm{c}$ & $26.9 \mathrm{a}-\mathrm{c}$ & $45.2 \mathrm{~g}-\mathrm{m}$ & $47.3 \mathrm{f}-1$ & 46.3h-1 & $89.6 \mathrm{f}-1$ & $136.5 \mathrm{c}-\mathrm{f}$ & $113.1 \mathrm{~d}-\mathrm{j}$ & $31.6 \mathrm{~b}-\mathrm{g}$ & $30.3 \mathrm{a}-\mathrm{i}$ & $31.0 \mathrm{~b}-\mathrm{f}$ & $28.3 \mathrm{e}-\mathrm{i}$ & $41.3 \mathrm{c}-\mathrm{f}$ & $34.8 \mathrm{~d}-\mathrm{h}$ \\
\hline Royal & $22.3 \mathrm{c}-\mathrm{g}$ & $24.6 \mathrm{a}-\mathrm{g}$ & $23.5 \mathrm{c}-\mathrm{h}$ & $45.2 \mathrm{~g}-\mathrm{m}$ & $47.7 \mathrm{f}-1$ & $46.5 \mathrm{~g}-\mathrm{k}$ & $77.9 \mathrm{~g}-\mathrm{n}$ & $125.5 \mathrm{~d}-\mathrm{i}$ & $101.7 \mathrm{f}-\mathrm{l}$ & $30.4 c-j$ & $30.5 \mathrm{a}-\mathrm{i}$ & $30.5 \mathrm{c}-\mathrm{g}$ & $23.8 \mathrm{f}-1$ & $38.3 \mathrm{c}-\mathrm{g}$ & $31.0 \mathrm{e}-\mathrm{j}$ \\
\hline Gila & $25.4 \mathrm{~b}-\mathrm{d}$ & $27.8 \mathrm{a}-\mathrm{c}$ & $26.6 \mathrm{a}-\mathrm{d}$ & $47.9 \mathrm{c}-\mathrm{k}$ & $48.2 \mathrm{e}-1$ & $48.1 \mathrm{f}-\mathrm{i}$ & $168.9 \mathrm{~b}$ & $192.0 \mathrm{ab}$ & $180.5 \mathrm{~b}$ & $29.5 \mathrm{e}-1$ & $29.8 \mathrm{a}-\mathrm{i}$ & $29.6 c-j$ & $49.8 \mathrm{~b}$ & $57.1 \mathrm{ab}$ & $53.5 \mathrm{~b}$ \\
\hline Yuyao & 19.4 e-j & $28.1 \mathrm{a}-\mathrm{c}$ & $23.7 \mathrm{c}-\mathrm{g}$ & $52.9 \mathrm{a}-\mathrm{c}$ & $49.8 c-j$ & $51.4 \mathrm{~b}-\mathrm{d}$ & $95.9 \mathrm{e}-\mathrm{k}$ & $127.5 \mathrm{~d}-\mathrm{i}$ & 111.7 e-j & $26.4 \mathrm{k}-\mathrm{o}$ & 27.0 e-m & $26.7 \mathrm{k}-\mathrm{p}$ & $25.2 \mathrm{e}-\mathrm{k}$ & $34.5 \mathrm{~d}-\mathrm{i}$ & 29.9 e-k \\
\hline Shufu & $8.5 \mathrm{n}$ & $8.0 \mathrm{k}-1$ & $8.2 \mathrm{q}$ & $52.0 \mathrm{a}-\mathrm{d}$ & $55.6 \mathrm{ab}$ & $53.8 \mathrm{a}-\mathrm{c}$ & $66.3 \mathrm{j}-\mathrm{p}$ & $57.6 \mathrm{~m}-\mathrm{o}$ & $62.0 \mathrm{o}-\mathrm{s}$ & $22.8 \mathrm{p}$ & $22.3 \mathrm{~m}$ & $22.6 \mathrm{r}$ & $15.1 \mathrm{k}-\mathrm{o}$ & $12.8 \mathrm{o}-\mathrm{q}$ & $14.0 \mathrm{p}-\mathrm{r}$ \\
\hline Huaxia & $12.91-\mathrm{n}$ & $13.8 \mathrm{j}-\mathrm{k}$ & $13.4 \mathrm{o}-\mathrm{p}$ & $50.3 \mathrm{~b}-\mathrm{g}$ & $49.7 \mathrm{~d}-\mathrm{j}$ & $50.0 \mathrm{~d}-\mathrm{g}$ & $46.8 \mathrm{~m}-\mathrm{r}$ & 64.9 1-o & $55.9 \mathrm{p}-\mathrm{s}$ & $26.6 \mathrm{k}-\mathrm{o}$ & $24.7 \mathrm{k}-\mathrm{m}$ & $25.7 \mathrm{n}-\mathrm{q}$ & $12.41-\mathrm{o}$ & $16.0 \mathrm{n}-\mathrm{q}$ & $14.2 \mathrm{p}-\mathrm{q}$ \\
\hline FO-2 & $19.1 \mathrm{f}-\mathrm{k}$ & $27.5 \mathrm{a}-\mathrm{d}$ & $23.3 c-j$ & $51.7 \mathrm{a}-\mathrm{e}$ & $56.4 \mathrm{a}$ & $54.1 \mathrm{a}-\mathrm{c}$ & $100.7 \mathrm{e}-\mathrm{k}$ & $154.7 \mathrm{~b}-\mathrm{e}$ & $127.7 \mathrm{c}-\mathrm{g}$ & $26.7 \mathrm{k}-\mathrm{o}$ & $25.0 \mathrm{j}-\mathrm{m}$ & $25.9 \mathrm{n}-\mathrm{q}$ & $26.9 \mathrm{e}-\mathrm{k}$ & $38.5 \mathrm{c}-\mathrm{g}$ & $32.7 \mathrm{e}-\mathrm{j}$ \\
\hline Gifford & $31.5 \mathrm{a}$ & $27.4 \mathrm{a}-\mathrm{d}$ & $29.5 \mathrm{a}$ & $53.5 \mathrm{a}-\mathrm{b}$ & $54.9 \mathrm{a}-\mathrm{c}$ & $54.2 \mathrm{ab}$ & $150.0 \mathrm{~b}-\mathrm{d}$ & $135.3 \mathrm{~d}-\mathrm{f}$ & $142.7 \mathrm{c}-\mathrm{d}$ & $29.2 \mathrm{f}-1$ & $23.7 \mathrm{k}-\mathrm{m}$ & $26.4 \mathrm{~m}-\mathrm{p}$ & $43.7 \mathrm{~b}-\mathrm{d}$ & $32.0 \mathrm{f}-\mathrm{k}$ & $37.9 \mathrm{~d}-\mathrm{e}$ \\
\hline
\end{tabular}

Means with identical letters in the same column are not statistically significant at the level of $p<0.01$. 
Reducing the hull content in safflower is one of the significant goals of breeding programs. In the study, Ziyang, Gifford, FO-2 and Shufu had the highest hull content in 2011 and 2012; and according to the average of both years. Besides, Yuyao and Saffire in 2011 and Yenice 5-38, AC Stirling and AC Sunset in 2012 were the genotypes with high hull content. Arizona SC III had the lowest hull content value in 2011,2012 , and the average of the both years $(25.0,26.7$, and $25.9 \%$, respectively) (Table 5). It was reported that the genes controlling the seed hull thickness in safflower, also control the secondary wall thickening in stem cells and anther dehiscence of flowers (structural sterility) by having a pleiotropic effect. Therefore, genotypes with a thin hull have both thin stems with low fertility (Weiss, 2000). Arizona SC III had the lowest hull content and the highest oil content whereas its seed and oil yields were the lowest among the study material. Rudra Naik et al. (2009) reported that the environment may affect the hull content; however, the vast majority of the variation for hull content was due to genetic factors.

The seed yields of the genotypes ranged from 17.0 to $208.8 \mathrm{~kg} \mathrm{da}^{-1}$ in 2011 , from 13.5 to $223.0 \mathrm{~kg} \mathrm{da}^{-1}$ in 2012 , and from 15.2 to $215.9 \mathrm{~kg} \mathrm{da}^{-1}$ for the average of both years. For combined yields of both years, the highest seed yield was determined in UC-1 but the lowest seed yield in Arizona SC III. Gila and Dincer 5-118 also had high seed yields (192.0 and $183.3 \mathrm{~kg} \mathrm{da}^{-1}$, respectively) in 2012 . Of the registered cultivars in Turkey, only Dincer 5-118 gave a higher seed yield in both years than Remzibey-05 and Yenice 5-38 (Table 5). The seed yield of a genotype in a given year might vary by light, water, precipitation, temperature, humidity, and nutrient competition (Koutroubas et al., 2004). Additive genes affect the inheritance of the seed yield along with the environmental factors therefore seed yield show low heritability (Ghongade et al., 1993; Reddy et al., 2004; Camas and Esendal, 2006; Erbas, 2012). In the world, 40-170 $\mathrm{kg} \mathrm{da}^{-1}$ of yield could be obtained from safflower under nonirrigated conditions, but up to $300 \mathrm{~kg} \mathrm{da}^{-1}$ of yield can be obtained under favorable cultivation conditions (Weiss, 2000). The yield obtained from different safflower genotypes under arid conditions in the studies performed in different ecological regions in Turkey ranged from 113.1 to $316.4 \mathrm{~kg} \mathrm{da}^{-1}$ under the conditions of Ankara (Kolsarici and Ekiz, 1983), from 45 to $170 \mathrm{~kg} \mathrm{da}^{-1}$ under the conditions of Antalya (Baydar and Turgut, 1992), from 84.9 to $125.5 \mathrm{~kg} \mathrm{da}$ under the conditions of Kahramanmaras (Atakan, 1992), from 207.7 to $339.7 \mathrm{~kg}$ $\mathrm{da}^{-1}$ under the conditions of Eskisehir (Celikoglu, 2004), from 77.4 to $167.8 \mathrm{~kg} \mathrm{da}{ }^{-1}$ under the conditions of Erzurum (Öztürk et al., 2008), and from 45.6 to $298.0 \mathrm{~kg}$ $\mathrm{da}^{-1}$ under the conditions of Samsun (Camas and Esendal, 2006).

Safflower has lower oil content than the other important oilseed crops (e.g. sunflower, rapeseed, peanut, and sesame). The oil contents of the genotypes varied between 22.6 and $33.8 \%$ on average in the study. The highest oil content was determined in Centennial (33.8\%), Oleic Leed (33.8\%), Arizona SC III (33.8\%), followed by Finch (33.3\%) Ole (32.2\%), S-517 (31.8\%), Enana $(31.5 \%)$ and Leed $(31.4 \%)$ based on the average of both years. The lowest oil content was recorded in Shufu in both years (Table 5). The oil content is known to vary between 25 and $40 \%$ in commercial safflower cultivars (Knowles, 1982). Johnson et al. (1999) reported that the oil content ranged from 13 to $46 \%$ among 797 safflower introductions and the mean oil content was $27.0 \%$ among 137 introductions originated from Mediterranean basin, encompassing the safflower genotypes of Turkey as well. Fernandez-Martinez et al. (1993) reported that 200 safflower accessions from 37 countries contained 20.1 to $40.0 \%$ oil. Besides, safflower lines whose oil content could increase up to $55.0 \%$ were developed through intensive breeding research (Rubis, 2001). Oil content is known to change depending on cultivar, soil characteristics, and climate. By evaluating the results of the present study, it can be stated that said results are in accordance with those of previously published reports. The results could also be used to confirm that spiny safflower cultivars contain more oil than spineless cultivars (Weiss, 2000).

The oil yield of the genotypes varied between 5.1 and $65.1 \mathrm{~kg} \mathrm{da}-1$ for the average of both years. With the highest seed yield, UC-1 had the highest oil yield followed by Gila (53.5 $\left.\mathrm{kg} \mathrm{da}^{-1}\right)$. Even though, Arizona SC III had the highest oil content, it had the lowest oil yield due to its low seed yield (Table 5). Omidi (2000) reported that oil yield was significantly and positively correlated with seed yield and oil yield would also increase with increase in seed yield. Ada (2013) reported that the oil yield was in the range of $20.9-57.9 \mathrm{~kg} \mathrm{da}^{-1}$ on average in the safflower genotypes under Konya conditions, while Beyyavas et al. (2011) reported that oil yield ranged from 24.2 to $54.3 \mathrm{~kg} \mathrm{da}^{-1}$ on average under Adryaman conditions. Since the genotypes displayed variations in seed yield and oil content in the present study, they also displayed a large variation in oil yield.

The fatty acid composition of the safflower genotypes is presented in Table 6 . There are differences in the fatty acid composition of the genotypes. However, when the genotypes were examined individually, there were no marked changes in the fatty acid compositions for both years. The palmitic acid content was in the range of 9.1$13.7 \%$ in 2011 and in the range of 9.2-12.9 in 2012. Stearic acid content ranged between 2.5 to $5.3 \%$ in 2011 and between 2.4 to $5.6 \%$ in 2012. The highest palmitic acid content was found in FO-2 in both years, whereas the lowest palmitic acid content was detected in Montola 2000 and S-517 (9.1\%) in 2011 and in Montola 2000 and Ole $(9.2 \%)$ in 2012 . The lowest stearic acid content was observed in PCA (2.5 and 3.1\%) and UC-1 (3.0 and 2.4\%) in 2011 and 2012, respectively. The highest stearic acid content was determined in Oker (5.3 and 5.6\%). Knowles (1989) reported that there was a recessive gene (st) which controlled the synthesis of the stearic acid and that many 
safflower cultivars with different stearic acid contents were available.

Table 6. Fatty acid composition of safflower genotypes in 2011 and 2012.

\begin{tabular}{|c|c|c|c|c|c|c|c|c|}
\hline & \multicolumn{4}{|c|}{2011} & \multicolumn{4}{|c|}{2012} \\
\hline & $\mathrm{C}_{16: 0}$ & $\mathrm{C}_{18: 0}$ & $\mathrm{C}_{18: 1}$ & $\mathrm{C}_{18: 2}$ & $\mathrm{C}_{16: 0}$ & $\mathrm{C}_{18: 0}$ & $\mathrm{C}_{18: 1}$ & $\mathrm{C}_{18: 2}$ \\
\hline Quiriego-88 & 10.9 & 4.2 & 18.5 & 65.7 & 11.0 & 4.2 & 20.1 & 64.6 \\
\hline Oker & 11.8 & 5.3 & 14.1 & 67.9 & 11.0 & 5.6 & 16.5 & 66.5 \\
\hline AC Sunset & 10.1 & 3.8 & 12.9 & 72.7 & 11.0 & 3.4 & 15.2 & 70.1 \\
\hline Enana & 11.0 & 4.0 & 24.5 & 60.1 & 10.0 & 3.8 & 27.3 & 58.0 \\
\hline Sahuaripa-88 & 11.3 & 3.5 & 17.9 & 66.8 & 12.0 & 3.4 & 21.0 & 63.4 \\
\hline Montola-2000 & 9.1 & 3.2 & 60.2 & 26.9 & 9.2 & 3.6 & 64.2 & 22.4 \\
\hline AC Stirling & 11.0 & 3.9 & 25.0 & 60.0 & 10.4 & 3.4 & 24.9 & 60.7 \\
\hline 4022 & 10.9 & 3.3 & 21.5 & 63.5 & 9.8 & 3.9 & 22.6 & 62.9 \\
\hline PCA & 10.5 & 2.5 & 13.2 & 73.2 & 10.2 & 3.1 & 15.3 & 70.8 \\
\hline Frio & 12.9 & 3.9 & 14.5 & 67.8 & 12.6 & 3.2 & 14.9 & 68.7 \\
\hline Oleic Leed & 9.2 & 3.3 & 46.6 & 40.3 & 9.8 & 3.9 & 47.9 & 37.9 \\
\hline Dincer 5-118 & 13.1 & 4.3 & 12.9 & 68.8 & 12.7 & 4.9 & 13.6 & 68.2 \\
\hline Yenice 5-38 & 11.1 & 4.2 & 12.3 & 71.8 & 10.5 & 3.9 & 14.3 & 71.0 \\
\hline Remzibey-05 & 12.3 & 3.3 & 17.9 & 68.0 & 11.5 & 3.6 & 18.9 & 65.7 \\
\hline Centennial & 12.8 & 5.1 & 15.2 & 63.5 & 12.6 & 5.3 & 15.7 & 66.4 \\
\hline S-517 & 9.1 & 4.0 & 68.3 & 18.0 & 10.2 & 3.9 & 71.6 & 14.2 \\
\hline Lesaf & 10.4 & 3.8 & 21.9 & 63.4 & 11.0 & 3.4 & 22.6 & 63.0 \\
\hline Saffire & 9.7 & 3.5 & 20.5 & 65.6 & 10.0 & 2.9 & 20.6 & 66.5 \\
\hline Rehbein & 9.9 & 3.8 & 13.6 & 72.1 & 10.3 & 4.6 & 14.0 & 70.4 \\
\hline Finch & 11.1 & 4.5 & 12.5 & 71.4 & 11.4 & 4.5 & 12.8 & 70.8 \\
\hline Leed & 9.2 & 3.1 & 12.3 & 74.7 & 9.5 & 3.6 & 13.0 & 73.9 \\
\hline Arizona SC III & 12.1 & 3.7 & 13.2 & 70.5 & 11.5 & 3.2 & 14.0 & 71.3 \\
\hline NO 55-633 & 10.4 & 3.5 & 65.4 & 19.6 & 11.0 & 2.9 & 68.1 & 17.3 \\
\hline Hartman & 9.9 & 4.2 & 22.5 & 62.8 & 10.3 & 3.1 & 23.7 & 62.9 \\
\hline Ziyang & 10.5 & 4.2 & 18.1 & 66.7 & 9.7 & 3.6 & 19.0 & 67.2 \\
\hline Ole & 8.6 & 3.2 & 62.9 & 24.7 & 9.2 & 3.8 & 63.9 & 23.1 \\
\hline UC-1 & 10.3 & 3.0 & 65.3 & 20.5 & 11.0 & 2.4 & 67.8 & 18.8 \\
\hline US-10 & 11.9 & 3.3 & 16.8 & 67.3 & 11.0 & 3.6 & 17.3 & 67.3 \\
\hline Rinconada & 10.6 & 3.6 & 17.2 & 68.0 & 12.0 & 3.1 & 18.1 & 66.8 \\
\hline $\mathrm{CH}-353$ & 12.1 & 4.3 & 12.8 & 70.3 & 11.6 & 3.5 & 15.2 & 69.7 \\
\hline Sidwill & 10.9 & 4.1 & 14.7 & 69.8 & 11.5 & 4.3 & 16.4 & 67.8 \\
\hline San Jose-89 & 11.4 & 5.1 & 15.0 & 68.0 & 12.4 & 4.6 & 17.2 & 65.1 \\
\hline Royal & 13.7 & 4.4 & 13.0 & 66.9 & 12.8 & 4.8 & 14.1 & 68.1 \\
\hline Gila & 12.8 & 4.0 & 14.4 & 67.9 & 11.9 & 3.5 & 15.0 & 69.4 \\
\hline Yuyao & 12.2 & 3.7 & 22.9 & 60.7 & 12.0 & 4.2 & 23.0 & 60.8 \\
\hline Shufu & 11.6 & 4.0 & 11.1 & 71.4 & 11.3 & 4.3 & 12.0 & 72.4 \\
\hline Huaxian & 12.6 & 3.9 & 13.3 & 69.6 & 11.4 & 3.4 & 13.7 & 70.9 \\
\hline FO-2 & 14.6 & 3.8 & 12.0 & 68.9 & 12.9 & 4.6 & 12.9 & 69.6 \\
\hline Gifford & 11.4 & 4.3 & 15.5 & 68.1 & 11.4 & 4.5 & 16.1 & 67.1 \\
\hline
\end{tabular}

$\mathrm{C}_{16: 0}$, palmitic acid; $\mathrm{C}_{18: 0}$, stearic acid; $\mathrm{C}_{18: 1}$, oleic acid; $\mathrm{C}_{18: 2}$, linoleic acid

Likewise, Johnson et al. (1999) reported that the palmitic acid ranged from 3.9 to $6.8 \%$ and the stearic acid from 1.1 to $4.5 \%$ among 797 safflower introductions. Even though the agriculture of linoleic acid rich safflower cultivars is widely carried out in the world, the interest in the cultivars with high oleic acid content has increased as oil has been displaying high stability in the recent years (Weiss, 2000). The oleic and linoleic acid contents of the genotypes showed wide variation. Variation for oleic acid was between 11.1 and $68.3 \%$ in 2011 and between 12.0 and $71.6 \%$ in 2012 . Linoleic acid content varied between 18.0 and $74.7 \%$ in 2011 and between 14.2 and $73.9 \%$ in 2012. Montola 2000, Ole, UC-1, NO 55-663 and S-517 were found to contain more than $60 \%$ oleic acid. On the other hand, the other genotypes contained more than $60 \%$ linoleic acid with the exception of Oleic Leed which contained moderate oleic and linoleic acids (46.6 and
$40.3 \%$ in 2011 and 47.9 and $37.9 \%$ in 2012 , respectively) (Table 7). Fernandez-Martinez et al. (1993) reported variation for oleic $(3.1-84.2 \%)$ and linoleic $(9.1-89.2 \%)$ acids was large among the 200 safflower accessions. Velasco and Fernandez-Martinez (1999) reported that the 132 accessions examined from the US safflower collection contained $5.8 \%$ palmitic acid $(3.4-10.2 \%), 2.2 \%$ stearic acid (0.8-9.9\%), $26.2 \%$ oleic acid (5.6-86.9\%), and $65.9 \%$ linoleic acid (7.1-88.7\%) on average. The oleic acid contents of the genotypes were relatively higher in 2012 than in 2011. Temperatures in the flowering and seed development periods (July-August) were higher in 2012. It is known that temperature fluctuations have significant effects on the fatty acid composition in safflower (Bartholomew, 1971). With the increased temperatures, there is a decrease in the activity of the enzymes catalyzing the synthesis of linoleic and linolenic acids 
from oleic acid. Therefore linoleic acid synthesis increase when plants grow in a cool climate, but synthesis of oleic acid increase during the hot growing conditions in safflowers (Röbbelen et al., 1989; Broun and Somerville, 1997). This gives rise to highly negative correlations between oleic and linoleic acids (>-0.90**) (Knowles, 1989; Erbas, 2012).

Due to its high tolerance for aridity and moderate tolerance to cold due to the strong root structure, safflower is one of the most likely alternative oilseed crops to be utilized in the arid and semi-arid agricultural lands of Turkey. The most important goals of safflower breeding include seed yield and oil content increase. The oil contents and seed yields of the available cultivars in Turkey are not at the desired level. To reach economic level of yield from safflower, new safflower cultivars should be introduced along with modern cultivation methods. Superior lines may be obtained from the transgressive likely segregation to occur upon the hybridization of genotypes with high oil or seed yield. Large variations in seed yield, oil yield, oil content, and fatty acid composition were observed in the present study, in which the adaptation performances of 39 safflower genotypes were examined in 2011 and 2012. UC-1 was detected to be the genotype with high values in terms of seed and oil yield. For oil content, Arizona SC III, Oleic Leed, Centennial, Finch, Ole, S-517, Enana and Leed were the genotypes with the highest oil content. The available cultivars in Turkey all have high linoleic acid content. Identification of new genotypes with high oleic acid content may allow modifying the fatty acid composition of the available cultivars with the breeding methods. Further studies to assess the adaptability of the genotypes examined in this paper might be necessary for other characters.

\section{LITERATURE CITED}

Ada, R. 2013. Cluster analysis and adaptation study for safflower genotypes. Bulgarian J. Agric. Sci. 19(1):103-109.

Alizadeh, K. 2005. Evaluation of safflower germplasm by some agronomic characteristics and their relationships on grain yield production in the cold dry land of Iran. Int. J. Agric. Bio. 7(3):389-391.

Anonymous. 2015a. FAO/STAD Database http://www.fao.org. (Accessed December 10, 2015).

Anonymous. 2015b. Turkey Statistical Institute http://www.tuik.gov.tr. (Accessed December 10, 2015).

Atakan, M. 1992. A study on yield and yield components of different varieties of safflower (Carthamus tinctorius L.) in Kahramanmaras condition. MS Thesis, University of Gaziantep, Kahramanmaras, Turkey.

Bartholomew, S.B. 1971. Temperature effects on the Fatty Acid Composition of Developing in Safflower (Carthamus tinctorius L.). MS Thesis, University of California, Davis.

Baydar, H and I. Turgut. 1992. Potential of winter growing of safflower (Carthamus tinctorius L.) in Antalya condition. University of Akdeniz J. Faculty Agric. 1(2):75-92.

Baydar, H. and S. Erbas. 2007. Safflower breeding for edible oil and biodiesel production in Turkey. $1^{\text {th }}$ National Oil Crops and Biodiesel Symposium. 28-31 May, Samsun, Turkey, 378-386.
Beyyavas, V. H. Haliloglu, O. Copur. and A. Yilmaz. 2011. Determination of seed yield and yield components of some safflower (Carthamus tinctorius L.) cultivars, lines and populations under the semi-arid conditions. African $\mathrm{J}$. Biotech. 10(4):527-534.

Broun, P. and C. Somerville. 1997. Accumulation of ricinoleic lesquerolic and densipolic acid an seeds to transgenic arabidopsis plants that Express a fatty acyl hdroxlasecDNA from castor bean. Plant Physiology 113:933-942.

Camas, N. and E. Esendal. 2006. Estimates of broad-sense heritability for seed yield and yield components of safflower (Carthamus tinctorius L.). Hereditas 143:55-57.

Camas, N. C. Cirak and E. Esendal. 2007. Seed yield oil content and fatty acids composition of safflower (Carthamus tinctorius L.) grown in northern Turkey conditions. University of Ondokuz Mayis, J. Faculty Agric. 22(1):98104.

Celikoglu, F. 2004. Determination of yield components of safflower lines in Eskisehir condition. MS Thesis, University of Ankara, Ankara, Turkey.

Channeshappa, M.G. 1980. Genetics of seed yield oil content and other quantitative character in safflower (Carthamus tinctorius L.). Mysore J. Agric. Sci. 14(3):463.

Deokar, A.B.S. and F.B. Patil. 1980. Analysis of Parameters of Variability in Some Indian Varieties of Safflower. Field Crop Abstracts 33(1): 536.

Emami, T. R. Naseri, H. Falahi and E. Kazemi. 2011. Response of yield, yield component and oil content of safflower (cv. Sina) to planting date and plant spacing on row in rained conditions of Western Iran. American-Euroasian J. Agric. Environ. Sci. 6:947-953.

Erbas, S. 2012. Development of safflower (Carthamus tinctorius L.) lines with high oil, oleic acid content and seed yield through hybridization breeding. PhD, University of Suleyman Demirel, Isparta, Turkey.

Eslam, B.P. H. Monrifar and T. Ghassemi. 2010. Evaluation of late season drought effects on seed and oil yields in spring safflower genotypes. Turk. J. Agric. For. 34:373-380.

Fernandez-Martinez, J., M. Del Rio and A. De Haro 1993. Survey of safflower (Carthamus tinctorius L.) germplasm for variants in fatty acid composition and seed characters. Euphytica 69:115-122.

Ghongade, R.A., P.A. Navale and B.P. Joshi. 1993. Estimates of variability parameters in safflower. J. Agril. Uni. 18:461462.

Johnson, R.C., J.W. Bergman and C.R. Flynn. 1999. Oil and meal characteristics of core and non-core safflower accessions from the USDA collection. Genet. Res. Crop Evol. 46:611-618.

Karademir, E., C. Karademir and R. Ekinci. 2007. Heritability of earliness, yield and fiber technological properties in cotton. University of Yüzüncü Yil J. Faculty Agric. Sci. 17(2):6772.

Kizil, S., Ö. Cakmak, S. Kirici and M. Inan. 2008. A comprehensive study on safflower (Carthamus tinctorius L.) in semi-arid conditions. Biotechnol. Biotechnol. Eq. 22(4):947-953

Knowles, P.F. 1982. Safflower Genetics and Breeding. In: Improvement of Oil-Seed and Industrial Crops by Induced Mutations. International Atomic Energy Agency, Vienna, 91-101.

Knowles, P.F. 1989. Safflower. In: Downey R. K. Röbbelen G. Ashri A. (eds) Oil Crops of the World. McGraw-Hill New York, 363-374.

Kolsarici, Ö. and E. Ekiz. 1983. Research on important agricultural properties of domestic and foreign origin 
safflower (Carthamus tinctorius L.) cultivars. University of Ankara J. Faculty Agric. 864:1-25.

Koutroubas, S.D., D.K. Papakosta and A. Doitsinis. 2004. Cultivar and seasonal effects on the contribution of preanthesis assimilates to safflower yield. Field Crops Res. 2:263-274.

Lakshmi Prayaga, P., P. Lakshmamma and P. Padmavthi. 2003. Characterization of safflower germplasm for physiological traits. Sesame and Safflower Newsletter 18:90-92.

Marquard, R. 1987. Qualitats analytic ImDienste der Ölpflanzenzüchtung. Fat. Sci. Technol. 89 95-99.

Mary, S.S. and A. Gopalan. 2006. Dissection of genetic attributes yield traits of fodder cowpea in $\mathrm{F}_{3}$ and $\mathrm{F}_{4}$. J. Appl. Sci. Res. 2(6):805-808.

Omidi, A.H. 2000. Correlation between traits and path analysis for grain and oil yield in spring safflower. Sesame and Safflower Newsletter 15:78-82.

Omidi, A.H., H. Khazaei and S. Hongbo. 2009. Variation for some important agronomic traits in 100 spring safflower (Carthamus tinctorius L.) genotypes. American-Euroasian J. Agric. Environ. Sci. 5(6):791-795.

Öztürk, E., H. Özer and T. Polat. 2008. Growth and yield of safflower genotypes grown under irrigated and non-irrigated conditions in a highland environment. Plant Soil Environ. 54(10): 453-460.

Pahlavani, M.H. 2005. Some technological and morphological characteristics of safflower from Iran. Asian Journal of Plant Sci. 4(3):234-237.

Pahlavani, M.H., G. Saeidi and A.F. Mirlohi. 2007. Genetic analysis of seed yield and oil content in safflower using $F_{1}$ and $F_{2}$ progenies of diallel crosses. Int. J. Plant Production 2:129-140.

Parameshwar, K.B. 2009. Stability of non-spiny breeding lines in safflower (Carthamus tinctorius L.). MS Thesis, Dharwad University of Agricultural Sciences, Dharwad, India.
Ramachandram, M. and J.V. Goud. 1981. Genetic analysis of seed yield oil content and their components in safflower (Carthamus tinctorius L.). Theor. Appl. Genet. 60:191-195.

Reddy, M.V.S., P. Chand, B. Vidyadhar and I.S.L. Devi. 2004. Estimation of genetic parameters for yield and its component in $\mathrm{F}_{4}$ generation of safflower (Carthamus tinctorius L.). Prog. Agric. 4(1):16-18.

Röbbelen, G., R.K. Downey and A. Ashri. 1989. Oil Crops of the World. McGraw Hill Books, USA.

Rowell, D.R. 1996. Soil science: methods and applications. Harlow Longman

Rubis, D.D. 2001. Developing new characteristics during 50 years of safflower breeding. In Proceedings of the $\mathrm{V}^{\text {th }}$ International Safflower Conference, 23-27 July 2001, Sidney, Austria.

Rudra Naik, V., G.M. Bentur, P.M. Salimath and K.G. Parameshwarappa. 2009. Introgression of non-spiny and high oil content in adapted generations of safflower (Carthamus tinctorius L.). Karnataka J. Agric. Sci. 22(1):3943.

Safavi, S.A., S.S. Pourdad, S.M. Safavi and A.S. Safavi. 2011. Heritability and genetic gain of some morphological traits in safflower (Carthamus tinctorius L.). American J. Sci. Res. 17:14-18.

SAS Institute, 1999. INC SAS/STAT User's Guide Release 7.0 Cary NC, USA.

Singh, V., N.M. Kolekar and N. Nimbkar. 2008. Breeding strategy for improvement of flower and seed yields in safflower. VII ${ }^{\text {th }}$ International Safflower Conference, New South Wales, Australia.

Velasco, L. and J.M. Fernandez-Martinez . 1999. Screening for low saturated fatty acids in safflower. Sesame and safflower newsletter, Cordoba, Spain.

Weiss, E.A. 2000. Oilseed Crops II ${ }^{\text {nd }}$ Edition Blackwell Sci. Ltd. 364 pages, Victoria, Australia. 